\title{
Low- and High-Pathogenic Avian Influenza H5 and H7 Spread Risk Assessment Within and Between Australian Commercial Chicken Farms
}

\author{
Angela Bullanday Scott ${ }^{1}$, Jenny-Ann L. M. L. Toribio', Mini Singh', Peter Groves', \\ Belinda Barnes ${ }^{2}$, Kathryn Glass ${ }^{3}$, Barbara Moloney ${ }^{4}$, Amanda Black ${ }^{4}$ \\ and Marta Hernandez-Jover ${ }^{5,6 *}$
}

'Sydney School of Veterinary Science, Faculty of Science, University of Sydney, Sydney, NSW, Australia, ${ }^{2}$ Quantitative Sciences, Department of Agriculture and Water Resources, Canberra, ACT, Australia, ${ }^{3}$ College of Medicine, Biology and Environment, Australian National University, Canberra, ACT, Australia, ${ }^{4} \mathrm{New}$ South Wales Department of Primary Industries, Orange, NSW, Australia, ${ }^{5}$ Graham Centre for Agricultural Innovation, School of Animal and Veterinary Sciences, Charles Sturt University and New South Wales Department of Primary Industries, Wagga Wagga, NSW, Australia, ${ }^{6}$ School of Animal and Veterinary Sciences, Charles Sturt University, Wagga Wagga, NSW, Australia

OPEN ACCESS

Edited by: Irene Iglesias, CENTER FOR ANIMAL HEALTH RESEARCH, INIA, Spain

Reviewed by:

Fernanda Dorea,

National Veterinary Institute, Sweden Marius Gilbert, Université libre de Bruxelles, Belgium

${ }^{*}$ Correspondence: Marta Hernandez-Jover mhernandez-jover@csu.edu.au

Specialty section: This article was submitted to Veterinary Epidemiology and Economics,

a section of the journal Frontiers in Veterinary Science

Received: 29 November 2017 Accepted: 14 March 2018

Published: 09 April 2018

Citation: Scott AB, Toribio J-ALML, Singh M, Groves P, Barnes B, Glass $K$ Moloney $B$, Black $A$ and HernandezJover M (2018) Low- and HighPathogenic Avian Influenza H5 and H7 Spread Risk Assessment Within and Between Australian Commercial Chicken Farms. Front. Vet. Sci. 5:63. doi: 10.3389/fvets.2018.00063
This study quantified and compared the probability of avian influenza (Al) spread within and between Australian commercial chicken farms via specified spread pathways using scenario tree mathematical modeling. Input values for the models were sourced from scientific literature, expert opinion, and a farm survey conducted during 2015 and 2016 on Australian commercial chicken farms located in New South Wales (NSW) and Queensland. Outputs from the models indicate that the probability of no establishment of infection in a shed is the most likely end-point after exposure and infection of low-pathogenic avian influenza (LPAl) in one chicken for all farm types (non-free range meat chicken, free range meat chicken, cage layer, barn layer, and free range layer farms). If LPAI infection is established in a shed, LPAI is more likely to spread to other sheds and beyond the index farm due to a relatively low probability of detection and reporting during LPAI infection compared to high-pathogenic avian influenza (HPAl) infection. Among farm types, the median probability for HPAl spread between sheds and between farms is higher for layer farms $(0.0019,0.0016$, and 0.0031 for cage, barn, and free range layer, respectively) than meat chicken farms (0.00025 and 0.00043 for barn and free range meat chicken, respectively) due to a higher probability of mutation in layer birds, which relates to their longer production cycle. The pathway of LPAI spread between sheds with the highest average median probability was spread via equipment $(0.015 ; 5-95 \%, 0.0058-0.036)$ and for HPAl spread between farms, the pathway with the highest average median probability was spread via egg trays $\left(3.70 \times 10^{-5} ; 5-95 \%\right.$, $\left.1.47 \times 10^{-6}-0.00034\right)$. As the spread model did not explicitly consider volume and frequency of the spread pathways, these results provide a comparison of spread probabilities per pathway. These findings highlight the importance of performing biosecurity practices to limit spread of the Al virus. The models can be updated as new information on the mechanisms of the Al virus and on the volume and frequency of movements shed-to-shed and of movements between commercial chicken farms becomes available.

Keywords: avian influenza, Australia, commercial chickens, H5, H7, scenario trees, partial consequence assessment, spread 


\section{INTRODUCTION}

The risk of low-pathogenic avian influenza (LPAI) virus spread in Australia is initially dependent on the risk of exposure of commercial chicken farms in this country to LPAI, which has been quantified for New South Wales by Scott et al. (1). After exposure to the virus, the risk of spread is then dependent on infection of the chicken with the virus and establishment of the virus within the flock (2-4). Once established in one flock, LPAI spread within farms (between sheds) and between farms can occur. LPAI infection can be associated with no clinical signs but a range of clinical illness in birds including respiratory disease can also be seen, thereby leading to production losses and decreased welfare $(2,5)$. For infections with H5 and H7 LPAI viruses, with further virus spread and the subsequent increasing number of infected birds, there is a greater possibility of mutation of the virus to high-pathogenic avian influenza (HPAI). HPAI has very high morbidity and mortality rates in gallinaceous poultry (up to $100 \%)(5)$. If mutation does occur, the risk of HPAI spread within and between farms must then be considered.

Factors influencing the success of LPAI or HPAI spread depend heavily on biosecurity actions put into place on the farm. Previous modeling work suggest that bird pickup trucks and feed trucks that move between farms and human movements between sheds were pathways associated with the highest risk of spread of AI. Emphasis to ensure good biosecurity practices associated with these pathways, such as vehicle disinfection and footbaths, was therefore made $(6,7)$. The timeliness of detection of clinical signs of infected flocks by farmers also plays a significant role in limiting spread of the disease. If the appropriate authority figures are contacted by farmers promptly, management practices can be put into place to limit spread of the virus both within and between farms $(2,8)$. This is supported by several previous mathematical modeling studies that revealed a reduction in the probability of AI spread to other farms if detection and reporting occurs earlier rather than later in the outbreak and if the detection threshold is lowered or frequent sampling occurs on high-risk farms (9-11).

All seven HPAI outbreaks in Australia to date have had only commercial chicken farms as the index farms; including commercial layer or meat chicken farms, with two outbreaks involving meat chicken breeder farms. Four of the seven HPAI outbreaks involved spread from the index farm to affect the nearby farms $(12,13)$. In addition, surveillance found evidence of LPAI infection among duck farms in the vicinity for two of the seven HPAI outbreaks, suggesting initial LPAI spread with subsequent mutation $(14,15)$. The focus on commercial chicken farms in this study is due to the comparatively small threat posed by non-commercial chicken farms to the Australian poultry industry. There is limited contact between non-commercial and commercial chicken farms in Australia. In addition, AI detection on non-commercial chicken farms, as did occur with three of the 12 LPAI cases detected in this country to date, has little impact on the industry, market, and consumers due to the small number of birds to destroy (14-16).

The pathways of spread in the past Australian HPAI outbreaks were suspected based on epidemiological investigations; examples identified include common dead bird pick up and egg transport vehicles among the affected farms $(13,17,18)$. However, it is currently unknown for the Australian context which pathways are most likely to cause spread, whether particular farm types are at more risk of spread than others, and the influence biosecurity practices will have on spread. Thus there is a need to quantify and compare the probability of both LPAI and HPAI spread for all types of Australian commercial chicken enterprises, i.e., cage, barn, and free range systems of both layer and meat chicken farms. Further, there is a need to quantify the effect of on-farm preventive actions that can mitigate the risk and impact of future AI outbreak occurrences in Australia.

In response to these needs, the aim of this study was first to estimate the probability of infection and establishment of LPAI virus after one chicken is exposed to the virus using results obtained from Scott et al. (1). Then, potential pathways for LPAI and HPAI spread between sheds and farms on all types of Australian commercial chicken enterprises were identified. A partial consequence assessment was then performed to estimate and compare the probabilities of LPAI and of HPAI spread between sheds and farms with particular focus on the differences in spread via the investigated pathways, without explicit consideration of pathway volume and frequency as insufficient information was available to incorporate consideration of these in this study. Comparison of study results will inform understanding of the most influential pathways of spread of LPAI and HPAI, and of any differences between farm types if these exist. This new knowledge can direct thinking about on-farm biosecurity practices that can be put into place to reduce the potential for AI spread.

\section{MATERIALS AND METHODS}

\section{Risk Assessment Model}

The overall study used the World Organisation of Animal Health (OIE) risk analysis framework (19) to conduct an exposure and partial consequence assessment in relation to AI for Australian commercial chicken farms. The exposure assessment considered the potential pathways by which chickens situated in a commercial layer or meat chicken farm can be exposed to avian influenza (AI) virus from wild birds. This assessment can be found in the study by Scott et al. (1). The current study focused on a partial consequence assessment, where the risk of spread was determined but the level of consequences following spread was not measured. This assessment considered the pathways by which these viruses can spread between sheds on the same farm and from one farm to other farms. The probability of these pathways occurring was calculated. Such pathways were portrayed using scenario trees (20) and developed using Microsoft Excel (PC/ Windows 7, 2010). The probabilities were estimated using Monte Carlo stochastic simulation modeling using the program @RISK 7.0 (Palisade Corporation, USA). Each simulation consisted of 50,000 iterations sampled using the Latin hypercube method with a fixed random seed of one.

\section{Data Sources}

Most of the input values used in this model were parameterized using data collected from a survey on commercial chicken 
farms in Australia $(8,21)$. This study defined commercial layer farms as those with more than 1,000 birds, and commercial meat chicken farms as those with more than 25,000 birds. It involved a comprehensive on-farm interview with farmers including questions related to farm management, biosecurity practices, and wild bird presence. In addition, input values were also obtained from scientific literature. An expert opinion workshop was also held to obtain input values that were largely unknown or undescribed in the scientific literature (22).

\section{Survey on Commercial Layer and Meat Chicken Farms in the Sydney Basin Region and South East Queensland}

A survey was conducted from mid-2015 with on-farm interviews on 73 commercial chicken farms; nine cage layer, 9 barn layer, 25 free range layer, 15 non-free range meat chicken, and 15 free range meat chicken farms $(8,21)$. The farms were located in the Sydney basin region in New South Wales (NSW) and in South East Queensland. The Sydney basin region was selected due to the high concentration of both layer and meat chicken farms in this area. However, in this region, free range meat chicken farms are all owned by one of the two large privately owned meat chicken companies in Australia. Therefore, additional farm visits to South East Queensland were conducted to gain more representative data of privately owned meat chicken companies in Australia. The interviews with the farm manager or farm owner involved a comprehensive questionnaire with questions relating to biosecurity practices performed on farm, wild bird and animal presence, general farm information, and farm management. A greater proportion of layer farms and of free range farms were surveyed due to the greater perceived risk of AI occurrence on these farm types. Further details on the survey methodology, including the region and farm selection, questionnaire development, and conduct of the on-farm interviews can be found in the study by Scott et al. (21).

\section{Expert Opinion}

Due to many unknowns related to the AI virus, an expert elicitation process was conducted in late 2015 to help inform the parameters of mutation from LPAI to HPAI and farm-tofarm spread pathways; the shed-to-shed spread pathways were informed from a combination of scientific literature and the farm survey. The elicitation process used a modified Delphi technique to gather the information, based on a four-step elicitation process. The process involved the experts completing a questionnaire individually, followed by a discussion of the results at a workshop, and then a reassessment of the questionnaire answers after the workshop. A total of 10 experts who had varying levels of expertise related to the poultry industry, wild bird behavior, and AI virus characteristics, participated in the process. The experts were selected based on their experience in the Australian poultry industries including involvement in the management of HPAI outbreaks in Australia or overseas as well as knowledge on the AI virus and wild birds. The questionnaire included 39 probability questions, and experts were asked to provide a most likely, minimum and maximum estimates of the probabilities and their level of confidence on their estimates. Pert distributions were used to obtain individual expert estimates for each question. The second round of estimates for each question for all experts was then combined using a weighting factor depending on their respective level of expertise relevant to each question, in a discrete distribution. More details on the expert elicitation process and the outcomes of the study can be found in the study by Singh et al. (22).

\section{Statistical Analysis}

The statistical program JMP ${ }^{\circledR}$ was used (ㄷ 2012 SAS Institute Inc., Cary, NC, USA) to conduct one-way analysis of variance (ANOVA) to analyze the differences between the outcome probabilities from the models for different farm types. The outcome probabilities compared using ANOVA were the outcome probability from 1,000 iterations of each pathway endpoint of the spread scenario tree model simulation for each farm type with each iteration reflecting the situation for one farm at any point in time. A $p$-value of $<0.05$ was used to determine statistical significance in these analyses.

\section{Partial Consequence (Spread) Assessment}

The partial consequence assessment investigates the pathways of AI virus spread after one bird has been exposed to the virus at any point in time. It provides a comparison of spread probabilities between pathways; however, the volume and frequency of each pathway occurring were not explicitly considered. For shed-to-shed spread, there is consideration of the proportion of farms that perform or have these pathways present in combination with the survival of the virus on these pathways. For farm-to-farm spread, it was assumed that variation between pathways in volume and frequency and in virus survival was considered by experts. From the assumed LPAI exposure of one bird, spread first depends on infection of this bird, and this probability differs between direct or indirect exposure. In addition, spread depends on establishment of the virus within the shed after infection of one individual, which is influenced by the subtype of the virus. Both LPAI and HPAI spread are assessed, where the probability of $\mathrm{H} 5 / \mathrm{H} 7$ mutation from LPAI to HPAI is also considered after establishment within a flock. The end-points of this model are exclusive of one another and are as follows: (1) no establishment of the infection; (2) limited LPAI spread; (3) limited HPAI spread; (4) LPAI spread; and (5) HPAI spread.

Limited spread is defined as the spread that would occur even when infection is detected and reported by the farmer. In this situation, although it is assumed that control measures will be put into place to restrict further spread of the virus, spread prior to detection and reporting would be likely to occur due to the routine large volume of activities between both sheds and farms. Supporting this assumption, the number of days required for detection and reporting was estimated using an index function on Microsoft Excel, resulting in a time period of at least 70 days from infection of the first chicken with LPAI to establishment, detection 
and reporting by the farmer for all farm types. This estimation considered a reproduction number $(R)$ of 1.35 , the proportion of birds showing clinical signs, the shed size, and the percentage threshold for LPAI detection and reporting. The calculation of $\mathrm{R}$ and the proportion of birds showing clinical signs are presented in the description of the Establishment of LPAI after infection in one chicken node in the Supplementary Material. The shed size and percentage threshold for LPAI detection and reporting differ for each farm type and are described by Scott et al. (21). If there is no detection and reporting, the potential pathways by which LPAI and HPAI can spread between sheds and between farms are evaluated for each farm type.

The spread models used to estimate shed-to-shed and farmto-farm spread are two separate models and are independent of each other. The same input parameters are used in both models with the exception of the last node that considers the different pathways of spread, shed-to-shed and farm-tofarm. The five pathways for spread between sheds are shown in Figure 1 and the 12 pathways for spread between farms are shown in Figure 2, following the nodes "LPAI spread methods" and "HPAI spread methods". The input parameters used are described in Table $\mathbf{1}$ and a detailed description of the nodes is provided in the Supplementary Material. The majority of nodes apply to both LPAI and HPAI spread, with some specific to LPAI or HPAI spread only. The specific nodes for LPAI spread are LPAI spread methods shed-to-shed and LPAI spread methods farm-to-farm. The specific nodes for HPAI spread are HPAI clinical signs, detection, and reporting, HPAI spread methods shed-to-shed, and HPAI spread methods farm-to-farm. The probabilities of the different spread pathways were complementary to each other in the spread scenario tree models (e.g., the sum of the probabilities of all pathways occurring equaled one).

\section{Sensitivity Analysis}

The Advanced Sensitivity Analysis on the program @RISK 7.0 (Palisade Corporation, USA) was used to determine the effect of input parameters on the model outputs. The input values varied from 0 to 1 in thirds $(0,0.3,0.6,1)$. Each input value of interest was assessed in a simulation of 1,000 iterations while all other input values were fixed to their base value. The model outputs assessed were the probability of LPAI and HPAI spread between both sheds and farms per farm type.

The effect of the following inputs of LPAI and HPAI spread between sheds and farms were investigated: (1) Probability that the H5/H7 LPAI subtype will establish within the flock from one infected chicken (Prob_Establishment); (2) Probability that LPAI established within the flock will mutate to HPAI (Prob_Mutation); (3) Probability that the farmer will detect and report disease to appropriate officials during LPAI establishment (Prob_LPAI_Detection); (4) Probability that HPAI will produce clinical signs with the assumption that the probability of detection is extremely high (Prob_HPAI_Detection).

In addition, the impact of the probability of spread to another shed or farm through any of the pathways considered in this assessment, which is dependent to a high extent on the level of biosecurity implemented on farm, was also investigated. As the probabilities of the different spread pathways were complementary to each other in the spread scenario tree models, each pathway has the same influence on the probability of spread on the sensitivity analysis. As such, only one pathway probability is included in the sensitivity analysis and the generic term Prob_PathwaySpread is used.

\section{RESULTS}

\section{Probabilities of LPAI and HPAI Spread}

Results from the spread models provided the overall probabilities of no establishment of LPAI and of LPAI and HPAI limited spread and LPAI and HPAI spread between both sheds and farms, given one chicken is exposed to LPAI virus from one wild bird in Australia at any point of time. The results are summarized in Table 2 and Figure 3. The pathways involved in calculating these probabilities incorporated the probability of LPAI infection in a chicken after exposure and the probability that the virus is able to spread and establish among chickens within a shed. For all farm types, the most likely end-point after one chicken is exposed and infected with LPAI is no establishment. For each pathotype, the overall probabilities of spread are identical for each farm type between sheds and between farms. The results also show that for all farm types, the probability of limited LPAI spread is lower than that of limited HPAI spread; that LPAI spread is more likely to occur than limited LPAI spread; and that HPAI spread is less likely to occur than limited HPAI spread.

Low-pathogenic avian influenza and HPAI spread occur when the randomly selected values for the beta distribution for the probability of detection and reporting in the spread model are very low or zero. The probabilities of LPAI spread between sheds and farms, although low for all farms, were estimated to be highest in free range farms compared to other farm types. The model estimated a median probability of LPAI spread of 0.068 and 0.059 for free range meat chicken and layer farms, respectively. Among indoor farms, the probability (median; 5-95\%) of LPAI spread between sheds and farms is higher in barn meat chicken farms (0.037; 0.015-0.073) when compared to the indoor layer farm types; cage layer $(0.027 ; 0.0028-0.079)$ and barn layer $(0.026$; $0.0030-0.071)$. The probabilities of HPAI spread between sheds and farms are lower than that of LPAI spread for all farm types (Table 2).

\section{Probabilities of the Different Spread Pathways}

Results of the probability of LPAI and HPAI spread between sheds and farms are summarized in Figure 4, which presents the averages of the median, $5 \%$ and $95 \%$ probability values per pathway among all farm types and provides a comparison of relative probability of spread between pathways that does not explicitly consider the volume and frequency of each respective pathway occurring.

The pathways of spread between sheds were estimated using farm survey data to determine the proportion of farms that would perform or have specific practices or pathways for each farm type. This was combined with scientific literature to determine 


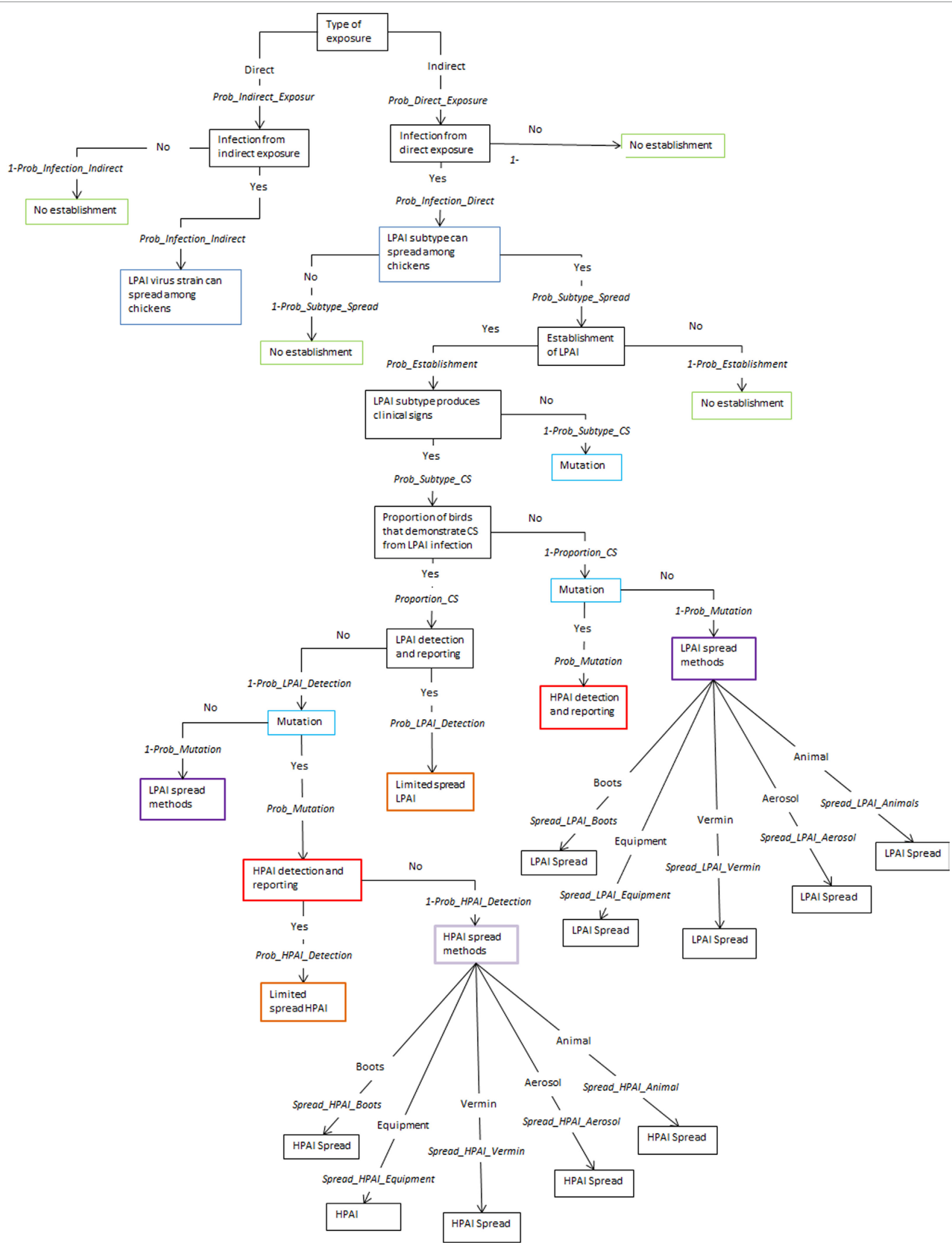

FIGURE 1 | Continued 
FIGURE 1 | Scenario tree representing the spread pathways of low-pathogenic and high-pathogenic avian influenza (LPAl and HPAI) viruses between sheds for Australian commercial layer and meat chicken farms. (Prob_Indirect_Exposure, probability of indirect exposure of LPAI virus to a commercial chicken; Prob_Direct_Exposure, probability of direct exposure of LPAI virus to a commercial chicken; Prob_Infection_Indirect, probability of infection of LPAI after indirect exposure; Prob_Infection_Direct, probability of infection of LPAI after direct exposure; Prob_Subtype_Spread, probability that the H5/H7 subtype that has infected a chicken is able to spread to other chickens; Prob_Establishment, probability that the H5/H7 LPAl subtype will establish within the flock from one infected chicken; Prob_Subtype_CS, probability that the LPAI H5/H7 subtype established within the flock is able to produce clinical signs within the flock; Proportion_CS, proportion of birds infected with LPAI that will produce clinical signs; Prob_Mutation, probability that LPAl established within the flock will mutate to HPAl; Prob_LPAI_Detection, probability that the farmer will detect and report disease to appropriate officials during LPAI establishment; Prob_HPAI_ Detection, probability that HPAI will produce clinical signs with the assumption that the probability of detection is extremely high; Spread_LPAI_Boots, probability that shed-to-shed spread of LPAI will occur via the movement of boots; Spread_LPAI_Equipment, probability that shed-to-shed spread of LPAI will occur via the movement of equipment; Spread_LPAI_Vermin, probability that shed-to-shed spread of LPAI will occur via the movement of vermin such as rats and insects; Spread_LPAI_Aerosol, probability that shed-to-shed spread of LPAI will occur via aerosol; Spread_LPAI_Animals, probability that shed-to-shed spread of LPAI will occur via the movement of other animals including pets; Spread_HPAI_Boots, probability that shed-to-shed spread of HPAI will occur via the movement of boots; Spread_HPAI_Equipment, probability that shed-to-shed spread of HPAI will occur via the movement of equipment; Spread_HPAI_Vermin, probability that shed-to-shed spread of HPAI will occurvia the movement of vermin such as rats and insects; Spread_HPAI_Aerosol, probability that shed-toshed spread of HPAI will occur via aerosol; Spread_HPAI_Animals, probability that shed-to-shed spread of HPAI will occur via the movement of other animals including pets).

the survival of the virus on each of these pathways, and similar volume and frequency for each pathway were assumed. The pathway of LPAI spread between sheds (Figure 4A) with the highest average median probability was spread via equipment $(0.015 ; 0.0058-0.036)$, followed by vermin $(0.010 ; 0.0028-0.023)$ and then boots $(0.0064 ; 0.00087-0.018)$. When the results of each farm type were assessed, the pathway of spread via equipment was the pathway with the highest median probability of LPAI spread between sheds for each farm type except free range layer farms. For this farm type, the pathway of LPAI spread between sheds with the highest median probability was spread via vermin (0.019;0.0022-0.041).

The pathway of HPAI spread between sheds (Figure 4B) with the highest average median probability was also spread via equipment $\left(5.76 \times 10^{-5} ; 1.90 \times 10^{-6}-0.00057\right)$. All farm types except free range layer farms had the pathway of spread via equipment as the pathway with the highest median probability of HPAI spread between sheds. For free range farms, the pathway with the highest median probability was spread via animals $\left(8.93 \times 10^{-5}\right.$; $2.57 \times 10^{-6}-0.001$ ) (data not shown in Figure 4).

The pathways of spread between farms were estimated from expert opinion which is assumed to have considered variation in volume and frequency and virus survival between pathways. The pathway of LPAI spread between farms (Figure 4C) with the highest average median probability was spread via bird pick up systems $(0.0072 ; 0.0019-0.02)$, followed by egg trays $(0.0059$; 0.00066-0.017). The latter applies to only layer farm types. When assessing each farm type on its own, the pathway with the highest median probability of LPAI spread between farms was bird pick up systems for both barn and free range meat chicken farm types. Spread via egg trays was the pathway with the highest median probability of LPAI spread between farms for all layer farms.

The pathway of HPAI spread between farms (Figure 4D) with the highest average median probability was spread via egg trays $\left(3.70 \times 10^{-5} ; 1.47 \times 10^{-6}-0.00034\right)$, followed by egg pallets $\left(2.07 \times 10^{-5} ; 7.86 \times 10^{-7}-0.00021\right)$, bird pick up systems $\left(1.57 \times 10^{-5} ; 4.83 \times 10^{-7}-0.00019\right)$, and farm workers $\left(1.41 \times 10^{-5}\right.$; $\left.4.43 \times 10^{-7}-0.00018\right)$. The former two apply to layer farms only. For individual farm types, and similar to that for LPAI, the pathway of HPAI spread between farms with the highest median probability was bird pick up systems for barn and free-range meat chicken farm types. Spread via egg trays was the pathway with the highest median probability of HPAI spread between farms for all layer farms.

\section{Spread Sensitivity Analysis}

Figure 5 shows the outputs of the spread sensitivity analysis, which depicts an example of one meat chicken or layer farm type per LPAI (Figures 5A,B) or HPAI (Figures 5C,D) spread between sheds and farms, as the sensitivity analysis outcomes were similar in proportional increase in value among all farm types. In addition, no difference on the spread sensitivity analyses for spread between sheds and spread between farms was observed.

According to the spread sensitivity analysis, the most influential parameter for LPAI spread between sheds and farms was the probability of establishment (Figures 5A,B). When the probability of establishment is increased to $100 \%$ (base value 0.47 for all farm types), there is an approximate 2.1 to 2.2 -fold increase on the probability of LPAI spread between sheds and farms for all farm types.

The probability of mutation was the most influential parameter affecting the probability of HPAI spread between sheds and farms for all farm types. When this probability is increased to $100 \%$ (base value $0.070,0.070,0.50,0.28,0.30$ for barn meat chicken, free range meat chicken, cage layer, barn layer and free range layer farms, respectively), there is at least a 3.5 -fold increase on the probability of HPAI spread between both sheds and farms for all farm types (Figures 5C,D). The influence of the probability of mutation is most substantial on meat chicken farm types where there is an approximate 17 -fold increase on the probability of HPAI spread between both sheds and farms within these farm types. The next most influential parameter on HPAI spread between sheds and farms was the probability of establishment where results obtained were similar to those seen with the LPAI spread sensitivity analysis described above.

The impact of the probability of detection on spread of LPAI and HPAI does not seem to be very significant. When this probability is increased to $100 \%$, there is only an approximate 0.05 -fold decrease on the probability of both LPAI (base value between 0.60 
and 0.70 for all farm types) and HPAI (base value 0.99 for all farm types) spread between sheds and farms for all farm types.

Investigation of the spread pathways revealed that when the probability of any of these pathways is increased to $100 \%$ (base values ranging from 0.00034 to 0.040 and $3.87 \times 10^{-7}$ and $8.83 \times 10^{-5}$ for LPAI and HPAI spread, respectively), there is an approximate 1.5 to 2 -fold increase on the probability of LPAI and HPAI spread between sheds and between farms for all farm types.

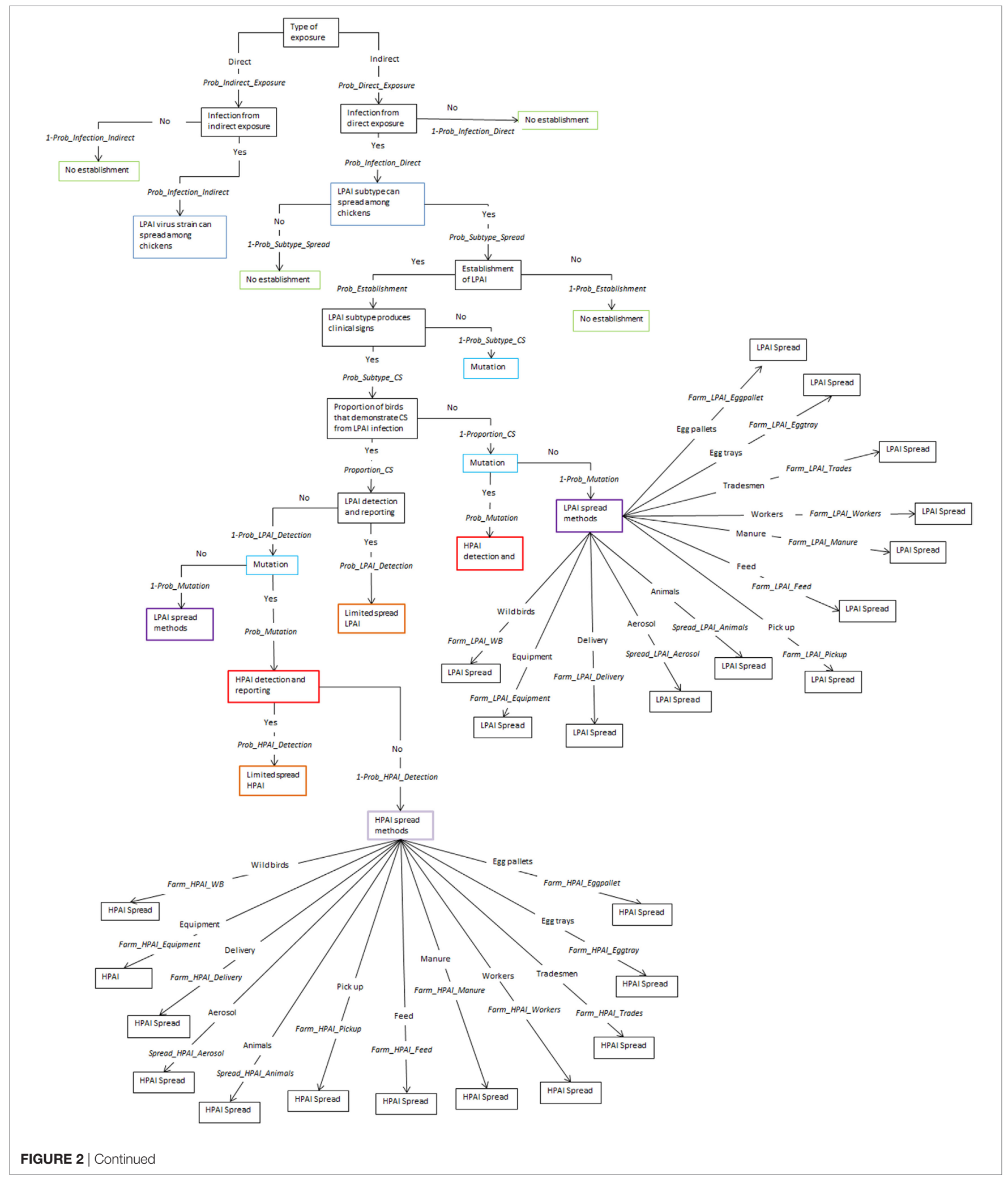


FIGURE 2 | Scenario tree representing the spread pathways of low-pathogenic and high-pathogenic avian influenza (LPAI and HPAI) viruses between farms for Australian commercial layer and meat chicken farms. (Prob_Indirect_Exposure, probability of indirect exposure of LPAI virus to a commercial chicken; Prob_Direct_ Exposure, probability of direct exposure of LPAI virus to a commercial chicken; Prob_Infection_Indirect, probability of infection of LPAI after indirect exposure; Prob_Infection_Direct, probability of infection of LPAI after direct exposure; Prob_Subtype_Spread, probability that the H5/H7 subtype that has infected a chicken is able to spread to other chickens; Prob_Establishment, probability that the H5/H7 LPAl subtype will establish within the flock from one infected chicken; Prob_ Subtype_CS, probability that the LPAI H5/H7 subtype established within the flock is able to produce clinical signs within the flock; Proportion_CS, proportion of birds infected with LPAI that will produce clinical signs; Prob_Mutation, probability that LPAl established within the flock will mutate to HPAl; Prob_LPAI_Detection, probability that the farmer will detect and report disease to appropriate officials during LPAl establishment; Prob_HPAI_Detection, probability that HPAI will produce clinical signs with the assumption that the probability of detection is extremely high; Farm_LPAI_Equipment, probability that farm-to-farm spread of LPAI will occur via the movement of equipment; Farm_LPAI_Aerosol, probability that farm-to-farm spread of LPAI will occur via aerosol; Farm_LPAI_Animals, probability that farm-to-farm spread of LPAI will occur via the movement of animals including both farm cats and dogs and vermin; Farm_LPAI_WB, probability that farm-to-farm spread of LPAI will occur via the movement of wild birds; Farm_LPAI_Delivery, probability that farm-to-farm spread of LPAI will occur via the movement of bird delivery transport vehicles; Farm_LPAI_Pickup, probability that farm-to-farm spread of LPAI will occur via the movement of dead and live bird pick up vehicles; Farm_LPAI_Feed, probability that farm-to-farm spread of LPAI will occur via the movement of feed delivery vehicles; Farm_LPAI_Manure, probability that farm-tofarm spread of LPAI will occur via the movement of manure collection systems; Farm_LPAI_Workers, probability that farm-to-farm spread of LPAI will occur via the movement of farm workers; Farm_LPAI_Tradesmen, probability that farm-to-farm spread of LPAI will occur via the movement of tradesmen such as plumbers and electricians; Farm_LPAI_Eggtray, probability that farm-to-farm spread of LPAI will occur via the movement of egg trays; Farm_LPAI_Eggpallet, probability that farm-to-farm spread of LPAI will occur via the movement of egg pallets; Farm_HPAI_Equipment, probability that farm-to-farm spread of HPAI will occur via the movement of equipment; Farm_HPAI_Aerosol, probability that farm-to-farm spread of HPAI will occur via aerosol; Farm_HPAI_Animals, probability that farm-to-farm spread of HPAI will occur via the movement of animals including both farm cats and dogs and vermin; Farm_HPAI_WB, probability that farm-to-farm spread of HPAI will occur via the movement of wild birds; Farm_HPAI_Delivery, probability that farm-to-farm spread of HPAI will occur via the movement of bird delivery transport vehicles; Farm_HPAl_Pickup, probability that farm-to-farm spread of HPAl will occur via the movement of dead and live bird pick up vehicles; Farm_HPAl_Feed, probability that farm-to-farm spread of HPAI will occur via the movement of feed delivery vehicles; Farm_HPAI_Manure, probability that farm-to-farm spread of HPAl will occur via the movement of manure collection systems; Farm_HPAI_Workers, probability that farm-to-farm spread of HPAI will occur via the movement of farm workers; Farm_HPAI_Tradesmen, probability that farm-to-farm spread of HPAl will occur via the movement of tradesmen such as plumbers and electricians; Farm_HPAI_Eggtray, probability that farm-to-farm spread of HPAI will occur via the movement of egg trays; Farm_HPAI_Eggpallet, probability that farm-to-farm spread of HPAl will occur via the movement of egg pallets).

This enabled evaluation of the change in probability of spread with implementation or presence of biosecurity practices that act on these spread pathways.

\section{DISCUSSION}

\section{The Probability of Spread}

The most likely pathway or outcome after one chicken is exposed and infected with LPAI is no establishment of the infection. This is supported by East et al. (38) where in all 17 samples tested positive for AI antibodies in the sentinel free-range flocks, there was no evidence of chicken-to-chicken transmission. However, these results contrast with work performed at the Australian Animal Health Laboratory (AAHL) where chickens inoculated and subsequently infected with various LPAI subtypes were placed in direct contact with other chickens. All chickens in direct contact with these infected chickens subsequently became infected (23). In addition, the spread model assumes only one chicken is exposed to the virus; it is unknown how many chickens are exposed to the virus and over what time period in an Australian context. Therefore, in order for model validation to occur, sampling of commercial chickens to determine their level of exposure to LPAI must be performed.

The overall probabilities of spread are identical for shed-toshed and farm-to-farm spread for each farm type and pathotype (presented in Table 2), and this is due to the only difference being the specific pathways of spread which are represented in the last node of the scenario tree (Figures 1 and 2). The probabilities of LPAI spread between sheds and farms are highest in free range farms. As previously mentioned, the spread model incorporates the probability of LPAI infection after the first bird has been exposed, where this probability is higher after direct exposure compared to indirect exposure. As such, the higher probability of LPAI spread in free range farms is due to exposure of the exposed bird on these farms to more likely be via direct pathways. Among non-free-range farms, the probability of LPAI spread, although similar, is slightly higher in barn meat chicken farms compared to the indoor layer farm types, due to the higher threshold of detection and reporting of sick and dead chickens in meat chicken farms compared to layer farms. The higher threshold provides more opportunity for the virus to spread before it is detected. In contrast, the probability of HPAI spread in meat chicken farms is lower than that of layer farms due to the short-lived nature of meat chicken birds leading to a lower probability of mutation in meat chicken birds compared to layer birds. This is reflected in expert opinion answers which informed the mutation parameter and gave a higher probability of mutation for layer farms compared to meat chicken farms (22).

Relative comparisons of these results to other countries can only be made for countries with similar LPAI and HPAI situations such as Australia, i.e., countries in which LPAI and HPAI are not endemic in poultry and HPAI is not endemic in wild birds. Countries in which HPAI H5N1 is endemic in poultry include Bangladesh, China, Egypt, India, Indonesia, and Vietnam (39). Similarly, comparisons should only be made to those countries that have effective protocols setup to deal with positive detections to limit spread. In Australia, this is written in the Australian Veterinary Emergency Plan (AUSVETPLAN) for avian influenza, which was developed and agreed upon by government and the poultry industry. In the United Kingdom (UK) and United States of America (USA), similar protocols are written in the Notifiable Avian Influenza Disease Control Strategy and HPAI Response Plan (The Red Book), respectively 
TABLE 1 | Nodes, parameter estimates, and input values used for the partial consequence assessment estimating the probability of spread of Avian Influenza (Al) viruses from flocks on both layer and meat commercial chicken farms in Australiaa.

Node Branch of Parameter estimates Input values sources

node

Input values

Data sources

\section{Parameters that apply to both LPAI and HPAI spread}

1. Type of Direct

exposure Indirect

\section{Infection from Yes}

direct exposure No

\section{Probability of infection from} direct exposure to Al virus in one chicken (Prob_Infection_Direct) Average of (probability of infection from intranasal infection from gastrointestinal inoculation + probability of animal) inoculation + probability of infection as a direct in-contact

Prob_Direct_Exposure

Exposure section Average of all direct exposure outputs from the three seasons of the respective farm type exposure scenario trees. of this study (1) The following values (median; 5-95\%) of Prob_Direct_Exposure for each farm type were:

Non-free range meat chicken $(0.24 ; 0.095-0.47)$

Free range meat chicken $(0.52 ; 0.28-0.76)$

Cage layer $(0.36 ; 0.14-0.60)$

Barn layer $(0.32 ; 0.10-0.59)$

Free range layer $(0.77 ; 0.60-0.86)$

Prob_Indirect_Exposure

1. Prob_Direct_Exposure

\section{Probability of infection from intranasal inoculation (PrIntranasal)}

Average LPAI H5N2 viral titers in tracheal swabs of Mallard ducks was 103.8 EID 50/ml over 6 days post inoculation

26/26 chickens inoculated via intranasal route with $10^{4.69}$ TCID 50/ml H9N2 LPAl became infected

16/18 chickens inoculated via intranasal route with $10^{3.69}$ TCID 50/ml H9N2 LPAl became infected

Therefore, 42 (s) of 46 (n) chickens become infected when inoculated via intranasal route with virus concentration similar to what is naturally excreted from upper respiratory tract from ducks

PrIntranasal $=$ Beta $(s+1, n-s+1)$ Probability of infection from gastrointestinal inoculation (PrGIT)

Average LPAI H5N2 viral titers in cloacal swabs of Mallard ducks was 102.04 EID 50/m over 5 days post inoculation

1/22 chickens inoculated via gastrointestinal route with 102.69 TCID50/ml H9N2 LPAl became infected In natural setting viral titers in duck feces will range considerably, therefore pert distribution used

PrGIT $=$ Pert $(0,1 / 22,1)$ Probability of infection as a direct in-contact animal (PrContact)

2 in-contact chickens placed directly in-contact with H5N2 LPAl infected chickens (n), 2 became infected (s) PrContact $=$ Beta $(s+1, n-s+1)$ Prob_Infection_Direct $=$ average (PrIntranasal; PrGIT; PrContact)

3. Infection Yes Probability of infection from Relative proportions of the following are taken by summing the two values and dividing each

from indirect No

exposure

value by the sum:

chicken (Prob_Infection_Indirect)

(Relative likelihood of aeroso

exposure $\times$ Probability of

infection from aerosol + Relative

likelihood of all other indirect

exposure $\times$ Probability of infection

from diluted gastrointestina

inoculation)
Yao et al. (3),

Selleck (23),

Webster et al. (24)
Exposure section

on this study (1)

Yao et al. (3),

Jonges et al. (25)
Average of all indirect exposure outputs via aerosol from the three seasons of the respective farm type exposure scenario tree (PropAerosol)

Average of all other indirect exposure outputs from the three seasons of the respective farm type ${ }^{a}$ exposure scenario tree (PropIndirect) Probability of infection from aerosol (PrAerosol)

Assume virus concentration in air in realistic scenarios is very low from wild birds

0 (s) of 10 (n) chickens exposed to aerosol virus concentration of 102.69 TCID50/ml H9N2 LPAl became infected

PrAerosol $=$ Beta $(s+1, n-s+1)$ Probability of infection from gastrointestinal inoculation (PrGIT) 1/22 chickens inoculated via gastrointestinal route with 102.69 TCID 50/ml H9N2 LPAl became infected 0/31 chickens inoculated via gastrointestinal route with $10^{1.69}$ TCID 50/ml H9N2 LPAl became infected Therefore, 1 (s) of $53(n)$ chickens become infected when inoculated via gastrointestinal route with diluted virus concentration

PrGIT $=$ Beta $(s+1, n-s+1)$ Prob_Infection_Indirect $=($ PropAerosol $\times$ PrAerosol $)+($ Proplndirect $\times$ PrGIT $)$ 


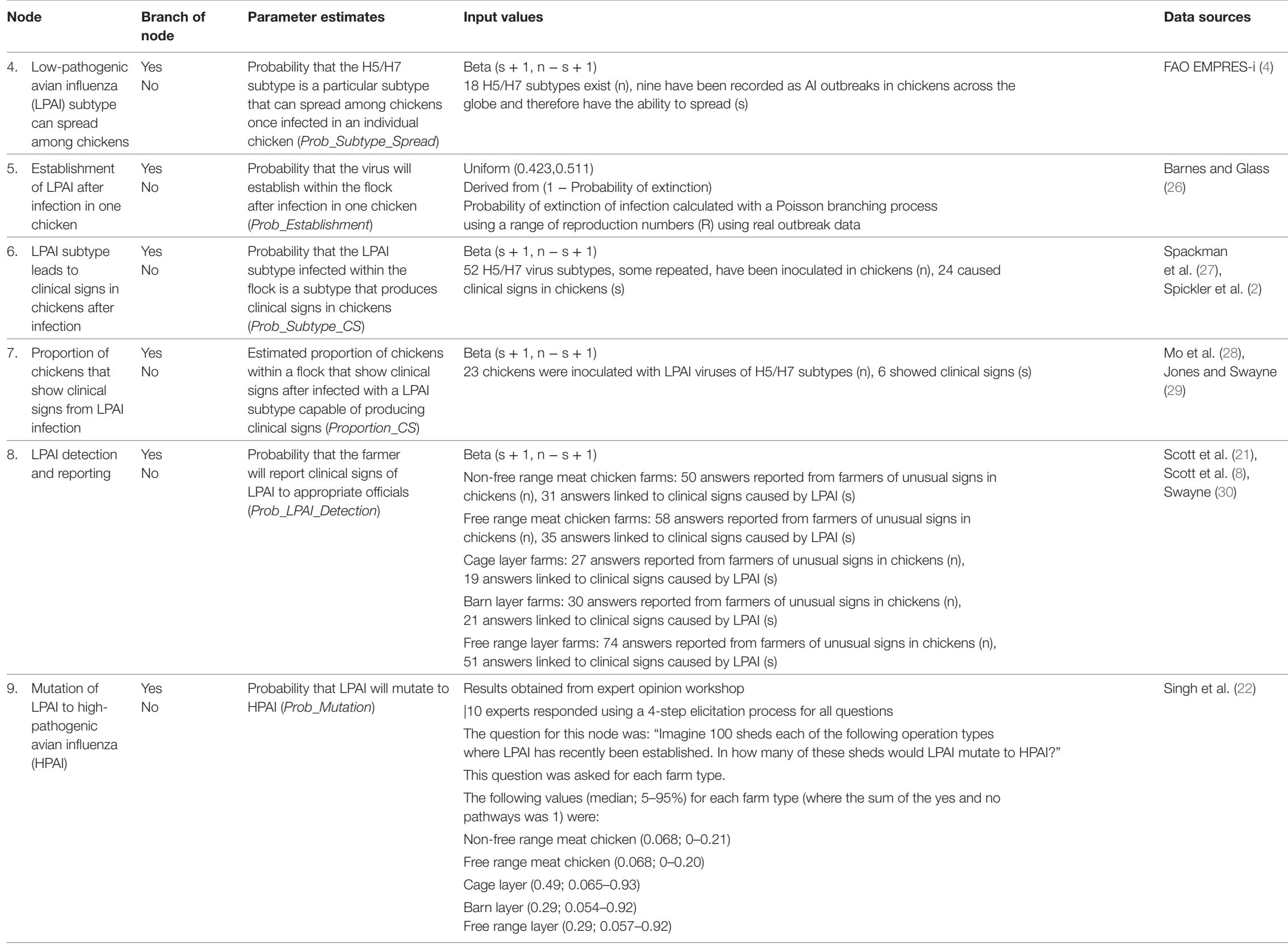




\section{Parameters that are specific to LPAI spread}

10. LPAl methods shed to shed Boots Equipment Vermin Aerosol

Probability that LPAI will spread between sheds via the following pathways: boots, equipment,

Pets
(Spread_LPAI_Boots; Spread LPAI_Equipment; Spread_LPAI Vermin; Spread_LPAI_Aerosol; Spread_LPAI_Animals,
Probability of $L P A I$ spread via boots

Beta $(s+1, n-s+1)$ of farm answers (PrBoots)

Non-free range meat chicken farms: 1/15 (s/n) answers did not use footbaths

Free range meat chicken farms: 1/15 (s/n) answers did not use footbaths

Cage layer farms: $7 / 9(\mathrm{~s} / \mathrm{n})$ answers did not use footbaths

Barn layer farms: $3 / 9(\mathrm{~s} / \mathrm{n})$ answers did not use footbaths

Free range layer farms: $6 / 25(\mathrm{~s} / \mathrm{n})$ answers did not use footbaths

Probability of virus presence on boots in one day is 1 as survival is longer than one day on this materia

Spread_LPAI_Boots $=($ PrBoots $) \times 1$

Probability of $L P A /$ spread via equipment

Beta ( $s+1, n-s+1)$ of farm answers (PrEquipment)

Non-free range meat chicken farms: 6/11 (s/n) answers do not clean equipment between sheds

Free range meat chicken farms: $9 / 9(\mathrm{~s} / \mathrm{n})$ answers do not clean equipment between sheds

Cage layer farms: $7 / 7(\mathrm{~s} / \mathrm{n})$ answers do not clean equipment between sheds

Barn layer farms: 6/7 (s/n) answers do not clean equipment between sheds

Free range layer farms: 2/23 (s/n) answers do not clean equipment between sheds

Probability of virus presence on equipment in one day is 1 as survival is longer than one day on this material

Spread_LPAI_Equipment $=($ PrEquipment $) \times 1$

Probability of $\angle P A l$ spread via vermin

Beta $(s+1, n-s+1)$ of farm answers (PrVermin)

Non-free range meat chicken farms: 24/30 (s/n) answers report vermin inside sheds

Free range meat chicken farms: $2 / 30(\mathrm{~s} / \mathrm{n})$ answers report vermin inside sheds

Cage layer farms: $17 / 18(\mathrm{~s} / \mathrm{n})$ answers report vermin inside sheds

Barn layer farms: 17/18 $(\mathrm{s} / \mathrm{n})$ answers report vermin inside sheds

Free range layer farms: 44/50 (s/n) answers report vermin inside sheds

Probability of virus presence/survival in vermin (SurvivalVermin):

Beta $(\mathrm{s}+1, \mathrm{n}-\mathrm{s}+1)$

0/12 (s/n) LPAl inoculated rats and 73/171 (s/n) LPAl inoculated fly pools were positive on virus isolation

Spread_LPAI_Vermin $=($ PrVermin $) \times($ SurvivalVermin $)$

Probability of $L P A$ I spread via aerosol

Beta $(s+1, n-s+1)$ of farm answers (PrAerosol)

Non-free range meat chicken farms: 15/15 (s/n) answers had sheds $<60 \mathrm{~m}$ from each other

Free range meat chicken farms: 15/15 (s/n) answers had sheds $<60 \mathrm{~m}$ from each other

Cage layer farms: 9/9 (s/n) answers had sheds $<60 \mathrm{~m}$ from each other

Barn layer farms: $9 / 9(\mathrm{~s} / \mathrm{n})$ answers had sheds $<60 \mathrm{~m}$ from each other

Free range layer farms: 25/25 (s/n) answers had sheds $<60 \mathrm{~m}$ from each other

Probability of virus presence/survival in air (SurvivalAerosol):

Beta $(s+1, n-s+1)$
Scott et al. (21);

Scott et al. (8);

Achenbach and

Bowen (31);

Nielsen et al. (32);

Tiwari et al. (33);

Jonges et al. (25):

Wood et al. (34) 
node

0/9 (s/n) air samples tested at $<60 \mathrm{~m}$ from LPAl infected chicken farms were positive for LPAl virus

Spread_LPAI_Aerosol $=($ PrAerosol) $\times($ SurvivalAerosol)

Probability of $L P A /$ spread via animals

Beta ( $s+1, n-s+1)$ of farm answers (PrAnimals)

Non-free range meat chicken farms: 0/15 (s/n) answers allow animals inside sheds

Free range meat chicken farms: 2/30 (s/n) answers allow animals inside sheds or range areas

Cage layer farms: $6 / 9(\mathrm{~s} / \mathrm{n})$ answers allow animals inside sheds

Barn layer farms: $1 / 9(\mathrm{~s} / \mathrm{n})$ answers allow animals inside sheds

Free range layer farms: $13 / 50(\mathrm{~s} / \mathrm{n})$ answers allow animals inside sheds or range areas

Probability of virus presence on other animals in one day is 1 as virus survival is longer than one

day on other animals

Spread_LPAI_Animals $=($ PrAnimals $) \times 1$

11. LPAl spread Aerosol Probability that LPAI will spread methods farm Infected wild between farms via the following to farm bird pathways: aerosol, infected

Animals (vermin wild bird going from one farm to and pets) another, other animals including Bird delivery vermin and pets, new bird

transport delivery transport, bird pick up Bird pick up transport both live and dead, transport (live feed delivery transport, manure and dead) collection, farm workers, trades Feed delivery people such as electricians and transport plumbers, shared equipment Manure between farms, egg trays ${ }^{b}$, egg collection $\quad$ pallets $^{\mathrm{b}}$ (Farm_LPAI_Aerosol;

Farm workers Farm_LPAI_WB; Farm_LPAI

Trades people Animals; Farm_LPAI_Delivery;

Shared Farm_LPAI_Pickup; Farm_LPAI

equipment Feed; Farm_LPAI_Manure;

Egg trays ${ }^{b} \quad$ Farm_LPAI_Workers; Farm

Egg pallets ${ }^{b} \quad$ LPAI_Trades; Farm_LPAI

Equipment; Farm_LPAI_Eggtray;

Farm_LPAI_Eggpallet)

\section{Parameters that are specific to HPAl spread}

12. HPAl clinical Yes

signs, detection No

and reporting
Results obtained from expert opinion workshop

Singh et al. (22)

10 experts responded using a 4-step elicitation process for all questions

The question for this node was: "Imagine 100 LPAl established (farm type)c farms. Realistically how many

of these will experience LPAI spread to at least one other chicken farm through each of the following pathways?"

The values for each pathway and farm type are present in the Supplementary Materia 
13. HPAl spread

Probability that HPAI will spread

Probability of HPAl spread via boots

between sheds via the following

Beta $(s+1, n-s+1)$ of farm answers (PrBoots)

methods shed Equipment

pathways: boots, equipment,

$\begin{array}{ll}\text { Vermin } & \text { pathways: boots, equipmen } \\ \text { Aerosol } & \text { vermin, aerosol or pets }\end{array}$

Pets (Spread_HPAI_Boots; Spread_

HPAI_Equipment; Spread_HPAI

Vermin; Spread_HPAl_Aeroso:

Non-free range meat chicken farms: 1/15 (s/n) answers did not use footbaths

Free range meat chicken farms: $1 / 15(\mathrm{~s} / \mathrm{n})$ answers did not use footbaths

Spread_HPAl_Animals)

Cage layer farms: $7 / 9(\mathrm{~s} / \mathrm{n})$ answers did not use footbaths

Barn layer farms: $3 / 9(\mathrm{~s} / \mathrm{n})$ answers did not use footbaths

Free range layer farms: 6/25 (s/n) answers did not use footbaths

Probability of virus presence on boots in one day is 1 as survival is longer than 1 day on this material

Spread_HPAI_Boots $=($ PrBoots $) \times 1$

Probability of HPAl spread via equipment

Beta ( $\mathrm{s}+1, \mathrm{n}-\mathrm{s}+1)$ of farm answers (PrEquipment)

Non-free range meat chicken farms: $6 / 1(s / n) 1$ answers do not clean equipment between sheds

Free range meat chicken farms: $9 / 9(\mathrm{~s} / \mathrm{n})$ answers do not clean equipment between sheds

Cage layer farms: $7 / 7(\mathrm{~s} / \mathrm{n})$ answers do not clean equipment between sheds

Barn layer farms: 6/7 (s/n) answers do not clean equipment between sheds

Free range layer farms: $2 / 23(\mathrm{~s} / \mathrm{n})$ answers do not clean equipment between sheds

Probability of virus presence on equipment in one day is 1 as survival is longer than one day on this material

Spread_HPAI_Equipment $=($ PrEquipment $) \times 1$

Probability of HPAl spread via vermin Beta ( $s+1, n-s+1$ ) of farm answers (PrVermin)

Non-free range meat chicken farms: $24 / 30(\mathrm{~s} / \mathrm{n})$ answers report vermin inside sheds

Free range meat chicken farms: 2/30 (s/n) answers report vermin inside sheds

Cage layer farms: 17/18 (s/n) answers report vermin inside sheds

Barn layer farms: 17/18 (s/n) answers report vermin inside sheds

Free range layer farms: $44 / 50(\mathrm{~s} / \mathrm{n})$ answers report vermin inside sheds

Probability of virus presence/survival in vermin (Survivalvermin):

Beta $(s+1, n-s+1)$

0/516 (s/n) HPAl exposed rats and 41/59 (s/n) HPAl inoculated flies were positive on virus isolation

Spread_HPAl_Vermin $=($ PrVermin $) \times($ SurvivalVermin $)$

Probability of HPAl spread via aerosol

Beta $(s+1, n-s+1)$ of farm answers (PrAerosol)

Non-free range meat chicken farms: 15/15 (s/n) answers had sheds $<150 \mathrm{~m}$ from each other

Free range meat chicken farms: 15/15 $(\mathrm{s} / \mathrm{n})$ answers had sheds $<150 \mathrm{~m}$ from each other

Cage layer farms: $9 / 9(\mathrm{~s} / \mathrm{n})$ answers had sheds $<150 \mathrm{~m}$ from each other

Barn layer farms: 9/9 $(\mathrm{s} / \mathrm{n})$ answers had sheds $<150 \mathrm{~m}$ from each other

Free range layer farms: 25/25 answers had sheds $<150 \mathrm{~m}$ from each other

Probability of virus presence/survival in air (SurvivalAerosol):

Beta $(s+1, n-s+1)$

22/90 (s/n) air samples tested at $<60 \mathrm{~m}$ from HPAl infected chicken farms were positive for HPAl virus

Spread_HPAI_Aerosol $=($ PrAerosol) $\times($ SurvivalAerosol $)$

Probability of HPAl spread via animals

Scott et al. (8),

Tiwari et al. (33),

Wood et al. (34),

Sawabe et al. (35)

Nettles et al. (36),

McCluskey (37)

(Continued) 
TABLE 1 | Continued

\begin{tabular}{|c|c|c|c|c|}
\hline Node & $\begin{array}{l}\text { Branch of } \\
\text { node }\end{array}$ & Parameter estimates & Input values & Data sources \\
\hline & & & $\begin{array}{l}\text { Beta }(s+1, n-s+1) \text { of farm answers (PrAnimals) } \\
\text { Non-free range meat chicken farms: } 0 / 15(s / n) \text { answers allow animals inside sheds } \\
\text { Free range meat chicken farms: } 2 / 30(s / n) \text { answers allow animals inside sheds } \\
\text { Cage layer farms: } 6 / 9(\mathrm{~s} / \mathrm{n}) \text { answers allow animals inside sheds } \\
\text { Barn layer farms: } 1 / 9(\mathrm{~s} / \mathrm{n}) \text { answers allow animals inside sheds } \\
\text { Free range layer farms: } 13 / 50(\mathrm{~s} / \mathrm{n}) \text { answers allow animals inside sheds } \\
\text { Probability of virus presence on other animals in one day is } 1 \text { as virus survival is longer than one day } \\
\text { on other animals } \\
\text { Spread_HPAI_Animals }=\text { (PrAnimals) } \times 1\end{array}$ & \\
\hline $\begin{array}{l}\text { 14. HPAl spread } \\
\text { methods farm } \\
\text { to farm }\end{array}$ & $\begin{array}{l}\text { Aerosol } \\
\text { Infected wild } \\
\text { bird } \\
\text { Animals (vermin } \\
\text { and pets) } \\
\text { Bird delivery } \\
\text { transport } \\
\text { Bird pick up } \\
\text { transport (live } \\
\text { and dead) } \\
\text { Feed delivery } \\
\text { transport } \\
\text { Manure } \\
\text { collection } \\
\text { Farm workers } \\
\text { Trades people } \\
\text { Shared } \\
\text { equipment } \\
\text { Egg trays } \\
\text { Egg pallets }^{\mathrm{b}}\end{array}$ & $\begin{array}{l}\text { Probability that HPAI will spread } \\
\text { between farms via the following } \\
\text { pathways: aerosol, infected } \\
\text { wild bird going from one farm to } \\
\text { another, other animals including } \\
\text { vermin and pets, new bird delivery } \\
\text { transport, bird pick up transport } \\
\text { both live and dead, feed delivery } \\
\text { transport, manure collection, } \\
\text { farm workers, trades people such } \\
\text { as electricians and plumbers, } \\
\text { shared equipment between farms, } \\
\text { egg trays }{ }^{b} \text {, egg pallets }{ }^{\circ} \text { (Farm_- } \\
\text { HPAI_Aerosol; Farm_HPAI_WB; } \\
\text { Farm_HPAI_Animals; Farm_HPAI_ } \\
\text { Delivery; Farm_HPAI_Pickup; } \\
\text { Farm_HPAI_Feed; Farm_HPAI_ } \\
\text { Manure; Farm_HPAI_Workers; } \\
\text { Farm_HPAI_Trades; Farm_HPAI_ } \\
\text { Equipment; Farm_HPAI_Eggtray; } \\
\text { Farm_HPAI_Eggpallet) }\end{array}$ & $\begin{array}{l}\text { Results obtained from expert opinion workshop } \\
10 \text { experts responded using a four-step elicitation process for all questions } \\
\text { The question for this node was: "Imagine } 100 \mathrm{HPAl} \text { established (farm type) farms. Realistically how } \\
\text { many of these will experience HPAl spread to at least one other chicken farm through each of the following } \\
\text { pathways?" } \\
\text { The values for each pathway and farm type are present in the Supplementary Material }\end{array}$ & Singh et al. (22) \\
\hline
\end{tabular}

${ }^{a} A$ spread scenario tree was performed for all farm types; non-free range meat chicken, free range meat chicken, cage layer, barn layer and free range layer.

'These pathways applied to layer farms only; cage layer, barn layer, and free range layer.

cThis question was asked for each farm type. 
TABLE 2 | Median (5 and 95 percentiles) probabilities of no establishment and of low-pathogenic avian influenza (LPAl) and high-pathogenic avian influenza (HPAI) spread and limited spread between sheds and farms for the commercial chicken farm types (barn meat chicken, free range meat chicken, cage layer, barn layer, and free range layer) after exposure of one chicken to LPAl from one wild bird in Australia.

\begin{tabular}{|c|c|c|c|c|}
\hline Farm type & Median & $5 \%$ & $95 \%$ & $\begin{array}{c}F \text { statistic (degrees of } \\
\text { freedom); } P \text {-value }\end{array}$ \\
\hline \multicolumn{5}{|l|}{ No establishment } \\
\hline Barn meat chicken & 0.96 & 0.92 & 0.98 & \multirow[t]{5}{*}{$F(4,4995)=990.03 ;<0.0001$} \\
\hline Free range meat chicken & 0.92 & 0.86 & 0.96 & \\
\hline Cage layer & 0.94 & 0.89 & 0.97 & \\
\hline Barn layer & 0.95 & 0.9 & 0.98 & \\
\hline Free range layer & 0.89 & 0.83 & 0.93 & \\
\hline \multicolumn{5}{|l|}{ Probability of LPAI spread } \\
\hline Barn meat chicken & 0.037 & 0.015 & 0.073 & \multirow[t]{5}{*}{$F(4,4995)=490.61 ;<0.0001$} \\
\hline Free range meat chicken & 0.068 & 0.033 & 0.12 & \\
\hline Cage layer & 0.027 & 0.0031 & 0.079 & \\
\hline Barn layer & 0.026 & 0.003 & 0.071 & \\
\hline Free range layer & 0.059 & 0.0071 & 0.12 & \\
\hline \multicolumn{5}{|l|}{ Probability of HPAI spread } \\
\hline Barn meat chicken & $2.47 \times 10^{-5}$ & 0 & 0.00025 & \multirow[t]{5}{*}{$F(4,4995)=164.01 ;<0.0001$} \\
\hline Free range meat chicken & $4.60 \times 10^{-5}$ & 0 & 0.00043 & \\
\hline Cage layer & 0.00022 & $1.01 \times 10^{-5}$ & 0.0019 & \\
\hline Barn layer & 0.00017 & $7.33 \times 10^{-6}$ & 0.0016 & \\
\hline Free range layer & 0.00037 & $1.68 \times 10^{-5}$ & 0.0031 & \\
\hline \multicolumn{5}{|c|}{ Probability of limited LPAI spread } \\
\hline Barn meat chicken & 0.0032 & 0.0011 & 0.008 & \multirow[t]{5}{*}{$F(4,4995)=515.67 ;<0.0001$} \\
\hline Free range meat chicken & 0.0058 & 0.0022 & 0.013 & \\
\hline Cage layer & 0.0048 & 0.0017 & 0.012 & \\
\hline Barn layer & 0.0044 & 0.0015 & 0.011 & \\
\hline Free range layer & 0.0092 & 0.004 & 0.019 & \\
\hline \multicolumn{5}{|c|}{ Probability of limited HPAl spread } \\
\hline Barn meat chicken & 0.0044 & 0.0012 & 0.013 & \multirow[t]{5}{*}{$F(4,4995)=624.38 ;<0.0001$} \\
\hline Free range meat chicken & 0.0084 & 0.0025 & 0.022 & \\
\hline Cage layer & 0.021 & 0.0044 & 0.068 & \\
\hline Barn layer & 0.016 & 0.0035 & 0.063 & \\
\hline Free range layer & 0.034 & 0.0087 & 0.11 & \\
\hline
\end{tabular}

(40-42). The UK experienced 11 HPAI outbreaks since 2006, all of which were effectively eradicated by destroying all birds on infected premises, comparable to the results of this study which indicate limited HPAI spread to occur more often than HPAI spread (43). However, the USA has experienced more extensive HPAI outbreaks involving dozens of farms, which cost over hundreds of millions of dollars to effectively eradicate. These include the HPAI outbreaks that occurred in Pennsylvania in 1983 and 1984, and the more recent HPAI outbreaks since 2014 that affected more than 10 USA states $(44,45)$. Suggested factors influencing these extensive HPAI outbreaks in the USA include poor biosecurity between farms, and high levels of exposure to AI virus in poultry farms in general, leading to numerous separate introduction and infection events in addition to spread between sheds and between farms (37).

\section{The Probability of Spread and the Probability of Limited Spread}

The spread models revealed that for all farm types, the probability of LPAI spread is greater than the probability of limited LPAI spread. This is because detection and reporting is less likely to occur following LPAI establishment and so control measures are less likely to put into place that will limit LPAI spread. In contrast, the spread models indicate that limited HPAI spread is more likely to occur than HPAI spread due to the high probability farmers will detect and report the changes in morbidity and mortality that follow HPAI establishment in a chicken flock. In general, there is limited information to determine if shed-toshed spread has occurred on Australian LPAI-infected farms. There is evidence that shed-to-shed spread may have occurred on two farms; specifically chickens in several sheds on one farm were seropositive to LPAI H6N2 in 2006 and LPAI H9N2 was detected in three sheds on a turkey farm in 2012 (46). However, it is also possible that independent introductions and infections occurred on the sheds of these farms instead of spread between sheds. There has only been one incursion to date with evidence of farm-to-farm LPAI spread in Australia; investigation of the 2012 H9N2 incursion identified a second infected turkey farm during trace back surveillance from the first turkey farm. This second turkey farm showed no clinical signs or increased mortality (14). As mentioned, it is very likely that LPAI detections in Australia are underreported due to these being non-clinical LPAI infections which provides credibility to the outputs of the spread model; that the probability of LPAI spread is greater than that of limited LPAI spread.

Most farms in Australia in which HPAI occurred had the virus spread to other sheds within the farm. However, all outbreaks 


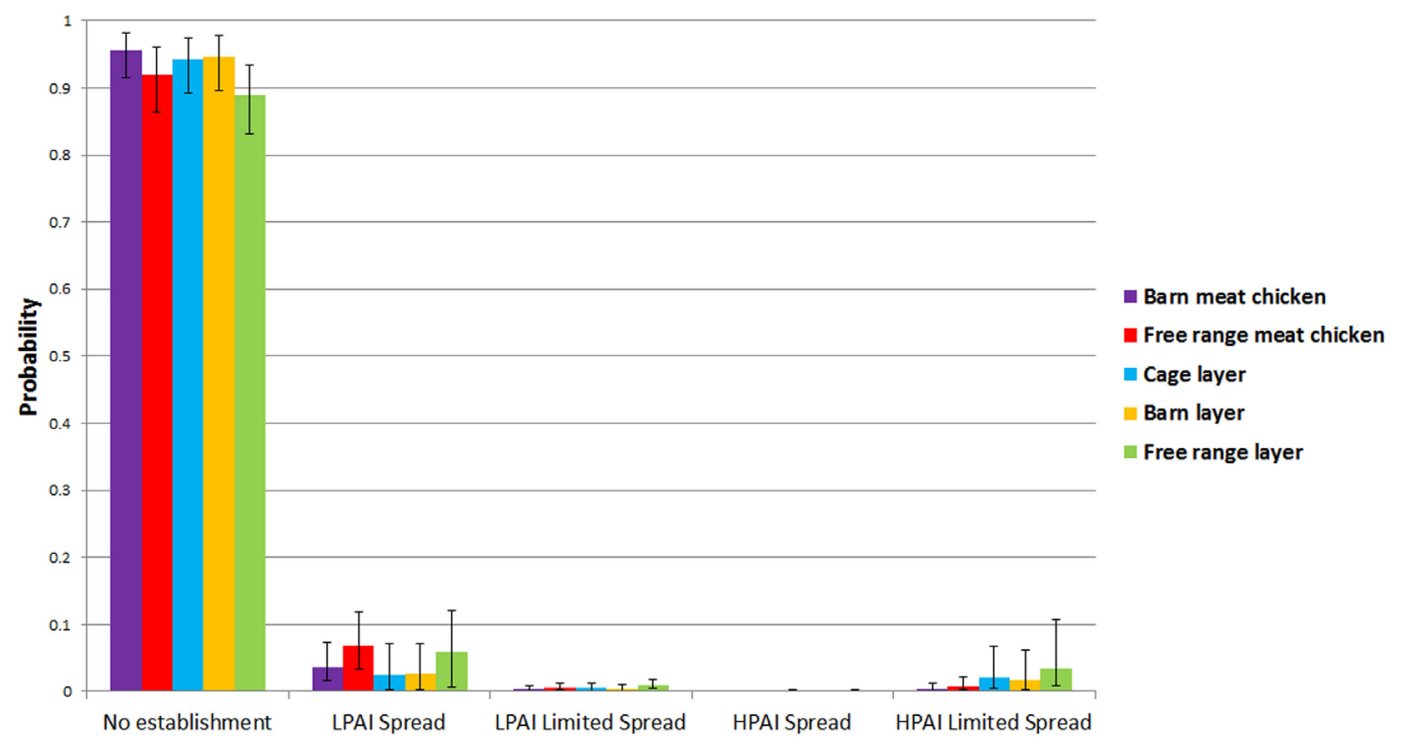

FIGURE 3 | Median (5 and 95 percentiles) probabilities of no establishment and of low-pathogenic avian influenza (LPAl) and high-pathogenic avian influenza (HPAl) spread and limited spread between sheds and farms for the commercial chicken farm types (barn meat chicken, free range meat chicken, cage layer, barn layer, free range layer) after one chicken is exposed to LPAI in Australia.

were effectively controlled via the stamping out procedure and resulting in limited farm-to-farm spread $(12,47,48)$. It is likely that the outputs of the spread model which indicate that the probability of HPAI spread is lower than that of limited HPAI spread reflect what has been experienced in Australia; this is easily seen with the farm-to-farm spread model.

\section{The Different Pathways of Spread}

The different pathways of LPAI and HPAI spread between sheds have differing probabilities. For LPAI spread between sheds, equipment and vermin were the most likely pathways and aerosol was the least likely pathway. For HPAI spread between sheds, equipment and boots were the most likely pathways and vermin was the least likely pathway. This is largely due to differences in the survival or detection of the virus reported in the literature relevant to these different pathways. LPAI spread via aerosol is regarded as an unlikely pathway in the literature, but detections of HPAI in air samples have been relatively frequently reported, particularly during the 2015 HPAI outbreaks in USA $(25,37)$. This is likely due to the higher levels of viral replication that occurs in the respiratory tract of birds with HPAI infection compared to LPAI infection (5). The relatively low probability of HPAI spread between sheds via vermin estimated in this study is likely due to how the input parameters in relation to this pathway were calculated. The input parameters were based on several studies where no virus isolation was obtained after exposure of vermin to AI viruses. It is generally been concluded that mice and rats do not play significant roles in the spread of AI virus but insects may $(31,32)$. In a study where a large number $(n=516)$ of samples were taken from rodents exposed to HPAI, no positive virus isolations were obtained (36). Similarly, a study where 12 rodents were inoculated with LPAI, no positive virus isolations were identified (31). The feeding of flies with LPAI and HPAI resulted in lower proportions of positive virus isolations from flies fed HPAI compared to LPAI $(32,35)$. The pathway of shedto-shed spread via vermin is possibly more significant for LPAI than HPAI.

When considering the results of this study, it must be remembered that the volume and frequency of the different spread pathways between both sheds and farms were not explicitly incorporated in the spread model. For shed-to-shed spread, these pathways were estimated using farm survey data in combination with scientific literature. The farm survey data were used to determine the proportion of farms that would perform or have specific practices or pathways for each farm type and scientific literature was used to determine the probability of survival of the virus on these pathways. It is known that there is a high frequency of daily movements between sheds and if incorporated in the model, may indicate that HPAI spread between sheds is morelikely than limited HPAI spread which would actually explain the high incidence of HPAI spread between sheds on farms affected by HPAI outbreaks in Australia $(12,47,48)$. This contrasts with the farm-to-farm spread pathways which were informed by expert opinion due to the lack of information in relation to these pathways. Expert understanding and answers of parameters influencing spread by each pathway can be assumed to have included consideration of the volume and frequency of occurrence and the survival of the virus for each pathway.

The output probabilities from the farm-to-farm spread model on the differing pathways of spread largely reflect the expert opinion answers where relatively higher probabilities of farm-to-farm spread were given to pickup trucks, egg trays, and egg pallets. These comparisons can be made from the model output results in Table 2 and the values in the Supplementary 
Material derived from expert opinion that were used to inform the pathways between farms (22). Expert estimates were largely influenced by the previous Australian HPAI outbreaks. An epidemiological investigation of the 2013 HPAI outbreak in Young, NSW suggested that the most likely route of spread of this virus to another farm was the contamination of cardboard egg trays (18). Similarly, a dead bird pick-up vehicle which visited multiple farms was the only identifiable link between farms that were affected by the 1997 HPAI outbreak in Tamworth, NSW (17). This compares with an expert opinion elicitation workshop published in 2011, which estimated the probability of HPAI spread between poultry farms to inform models simulating the transmission and control of HPAI epidemics in the Australian poultry industries. The results of this workshop showed that meat chicken pick up crews followed by slaughter crews, manure collection, and cardboard egg trays were rated as the most likely probabilities of transmission between farms (49). Differences observed between the two expert elicitations could be due to the time difference, as the 2012 and 2013 HPAI outbreaks had not yet occurred when the first expert elicitation was conducted.

\section{Spread Sensitivity Analysis}

There were no differences in values or trends on the spread sensitivity analyses of spread between sheds and spread between farms due to the identical structures of the models as described previously. The analyses revealed that the probability of establishment was an important influential parameter on the probability of LPAI and HPAI spread, as well as the probability of mutation on HPAI spread. Although influential, these parameters depend on virus properties and as such cannot be changed by human intervention, and there are large uncertainties associated with these mechanisms (50). Mutation from LPAI to HPAI has particularly large unknowns regarding its probability. A recent review of 42 HPAI outbreaks from 1959 to 2016, most of which involved chickens and turkeys as the initial species, concluded that emergence of HPAI can vary from a few days to a couple of years. It also considered that factors such as poultry age, size of the index farm, and type of farm management do not appear to contribute significantly to HPAI emergence (51). The expert opinion workshop also demonstrated very different estimated probabilities for mutation among the experts (22). The variation of $\mathrm{R}$, which was used to estimate the probability of establishment

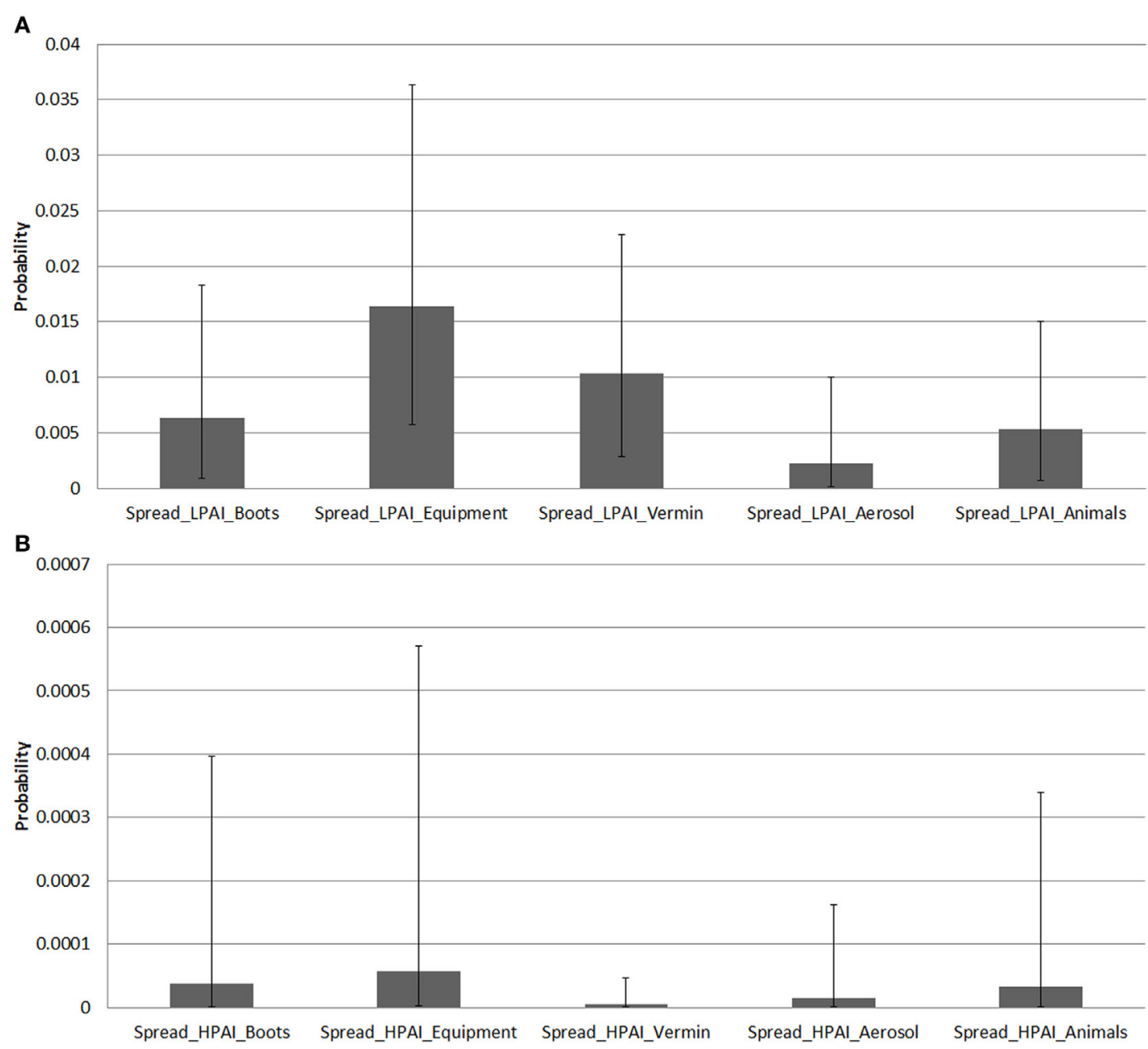

FIGURE 4 | Continued 


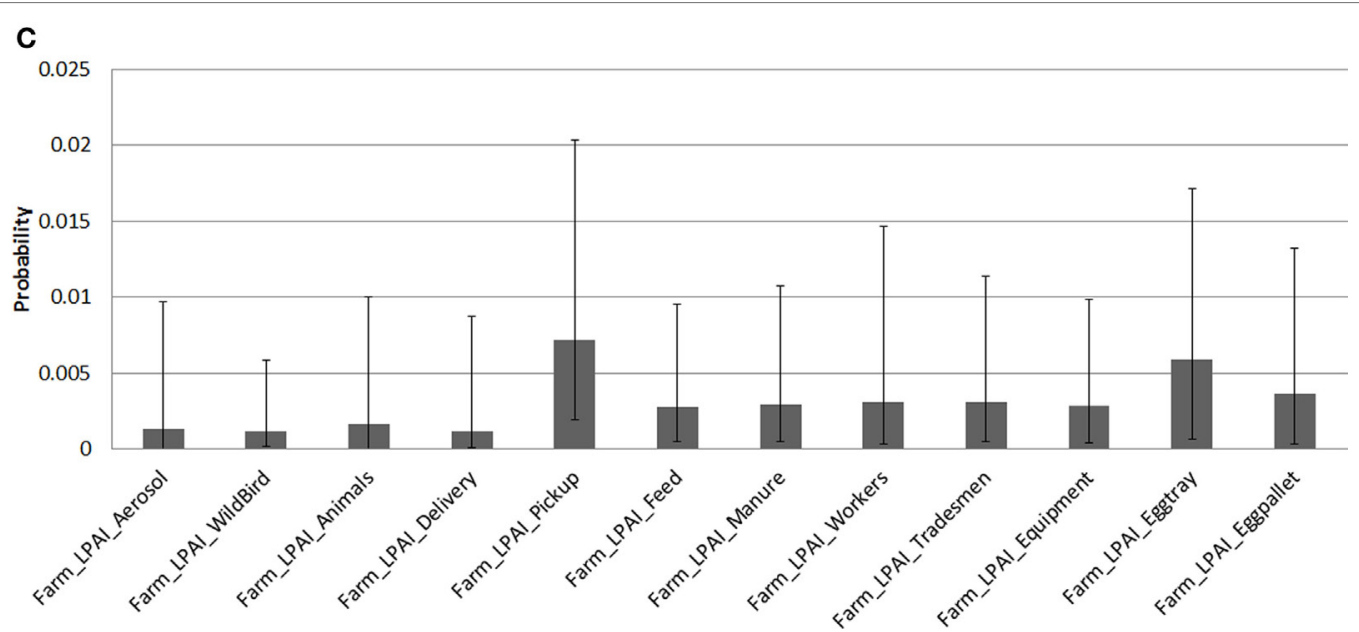

D

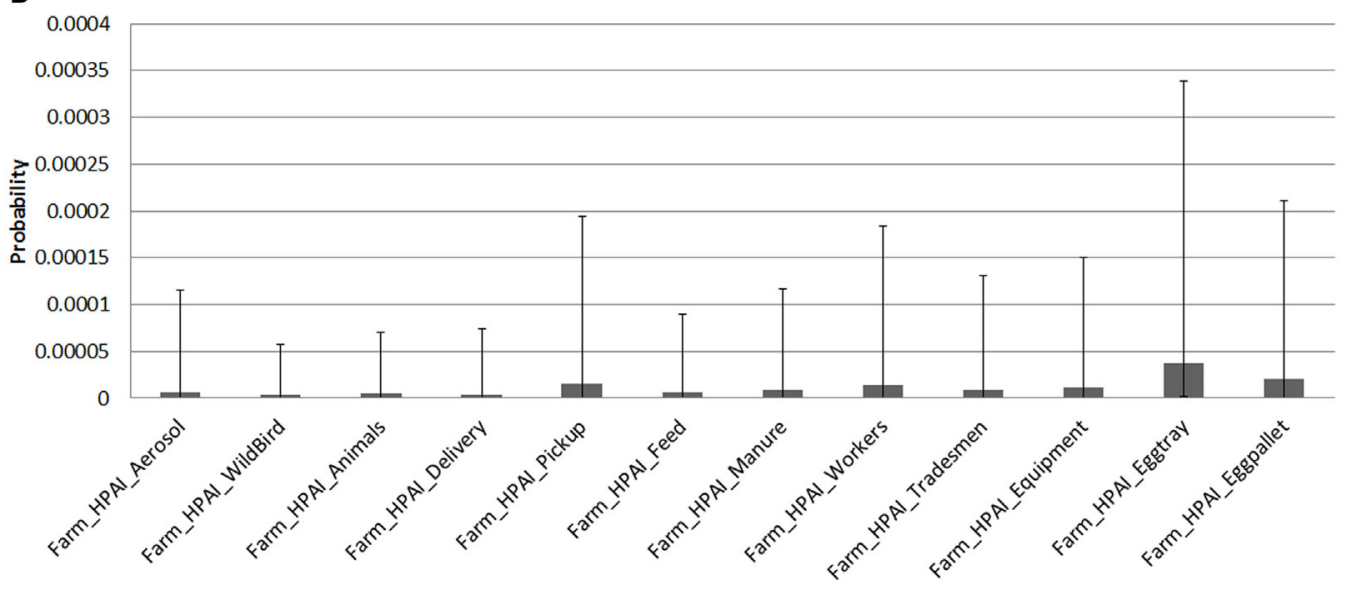

FIGURE 4 | Average median (5 and 95 percentiles) probabilities of low-pathogenic avian influenza (LPAl) and high-pathogenic avian influenza (HPAl) spread pathways between sheds and farms of the commercial chicken farm types (barn meat chicken, free range meat chicken, cage layer, barn layer, free range layer) after one chicken is exposed to LPAI in Australia. (A) Average median probabilities of LPAI spread pathways between sheds. Spread_LPAI_Boots, Probability that shed-toshed spread of LPAI will occur via the movement of boots; Spread_LPAI_Equipment, Probability that shed-to-shed spread of LPAI will occur via the movement of equipment; Spread_LPAI_Vermin, Probability that shed-to-shed spread of LPAI will occur via the movement of vermin such as rats and insects; Spread_LPAI_ Aerosol, Probability that shed-to-shed spread of LPAI will occur via aerosol; Spread_LPAI_Animals, Probability that shed-to-shed spread of LPAI will occur via the movement of other animals including farm cats and dogs. (B) Average median probabilities of HPAl spread pathways between sheds. Spread_HPAl_Boots, Probability that shed-to-shed spread of HPAI will occur via the movement of boots; Spread_HPAI_Equipment, Probability that shed-to-shed spread of HPAI will occur via the movement of equipment; Spread_HPAI_Vermin, Probability that shed-to-shed spread of HPAI will occur via the movement of vermin such as rats and insects; Spread_HPAI_Aerosol, Probability that shed-to-shed spread of HPAI will occur via aerosol; Spread_HPAl_Animals, Probability that shed-to-shed spread of HPAI will occur via the movement of other animals including farm cats and dogs. (C) Average median probabilities of LPAI spread pathways between farms. Farm_LPAI_ Equipment, Probability that farm-to-farm spread of LPAI will occur via the movement of equipment; Farm_LPAI_Aerosol, Probability that farm-to-farm spread of LPAI will occur via aerosol; Farm_LPAI_Animals, Probability that farm-to-farm spread of LPAI will occur via the movement of animals including both farm cats and dogs and vermin; Farm_LPAI_WB, Probability that farm-to-farm spread of LPAI will occur via the movement of wild birds; Farm_LPAI_Delivery, Probability that farm-to-farm spread of LPAI will occur via the movement of bird delivery transport vehicles; Farm_LPAI_Pickup, Probability that farm-to-farm spread of LPAI will occur via the movement of dead and live bird pick up vehicles; Farm_LPAI_Feed, Probability that farm-to-farm spread of LPAI will occur via the movement of feed delivery vehicles; Farm_LPAI_Manure, Probability that farm-to-farm spread of LPAI will occur via the movement of manure collection systems; Farm_LPAI_Workers, Probability that farm-to-farm spread of LPAI will occur via the movement of farm workers; Farm_LPAI_Tradesmen, Probability that farm-to-farm spread of LPAI will occur via the movement of tradesmen such as plumbers and electricians; Farm_LPAI_Eggtray, Probability that farm-to-farm spread of LPAI will occur via the movement of egg trays; Farm_LPAI_Eggpallet, Probability that farm-to-farm spread of LPAI will occur via the movement of egg pallets. (D) Average median probabilities of HPAI spread pathways between farms. Farm_HPAI_Equipment, Probability that farm-to-farm spread of HPAI will occur via the movement of equipment; Farm_HPAI_Aerosol, Probability that farm-to-farm spread of HPAI will occur via aerosol; Farm_HPAI_Animals, Probability that farm-to-farm spread of HPAI will occur via the movement of animals including both farm cats and dogs and vermin; Farm_HPAI_WB, Probability that farm-to-farm spread of HPAI will occur via the movement of wild birds; Farm_HPAI_Delivery, Probability that farm-to-farm spread of HPAI will occur via the movement of bird delivery transport vehicles; Farm_HPAI_Pickup, Probability that farm-to-farm spread of HPAl will occur via the movement of dead and live bird pick up vehicles; Farm_HPAI_Feed, Probability that farm-to-farm spread of HPAl will occur via the movement of feed delivery vehicles; Farm_HPAI_Manure, Probability that farm-to-farm spread of HPAl will occur via the movement of manure collection systems; Farm_HPAI_Workers, Probability that farm-to-farm spread of HPAI will occur via the movement of farm workers; Farm_HPAI_Tradesmen, Probability that farm-to-farm spread of HPAI will occur via the movement of tradesmen such as plumbers and electricians; Farm_HPAI_Eggtray, Probability that farm-to-farm spread of HPAI will occur via the movement of egg trays; Farm_HPAI_Eggpallet, Probability that farm-to-farm spread of HPAI will occur via the movement of egg pallets. 
in the current study, is significant in previous literature, even within the same pathotype and subtype $(49,52,53)$. As there is insufficient knowledge about mutation at present to in any way alter the likelihood of its occurrence, the control of LPAI and HPAI spread is therefore mainly reliant on on-farm biosecurity actions.

The detection and reporting parameters were found not to be a significantly influential parameter on the probability of LPAI and HPAI spread. This is supported by modeling work of Barnes and Glass (26), which demonstrated a high probability that a second shed is already infected with HPAI by the time initial infection is detected and reported using typical daily and weekly mortality rates for all farm types. In addition, the index formula described above used to calculate the number of days from infection in the first chicken to establishment, detection and reporting of LPAI also supports the small influence of detection, and reporting on the overall probability of spread. This formula revealed a long time period of at least 70 days for all farm types; within this time period, it is very possible that spread has already occurred to other sheds or farms due to the high level of movements between sheds and farms on all farm types $(54,55)$. This compares with previous modeling studies which revealed the high significance of detection and reporting in limiting spread of an AI outbreak. However, these studies assessed different but related factors to detection and reporting; including the impact of changing the detection threshold, performing frequent sampling of farms considered high risk, and ensuring prompt action after detection. In contrast, this study assumed a relatively fixed detection threshold based on farmer answers on unusual clinical signs, and therefore the changing parameter in the sensitivity analysis is simply a change in the proportion of farms that will detect and report at this relatively fixed detection threshold. Considerations to further evaluate the significance of detection and reporting are therefore described below.

The spread pathways on the scenario tree models were complementary to each other where the sum of all LPAI or HPAI spread pathway probabilities of one scenario tree model was one. Therefore, the spread sensitivity analysis could not accurately portray the effects of changing one spread pathway as this would result in complementary changes to the other spread pathways; each spread pathway had the same influence on the probability of spread. This was depicted as "Prob_PathwaySpread" which

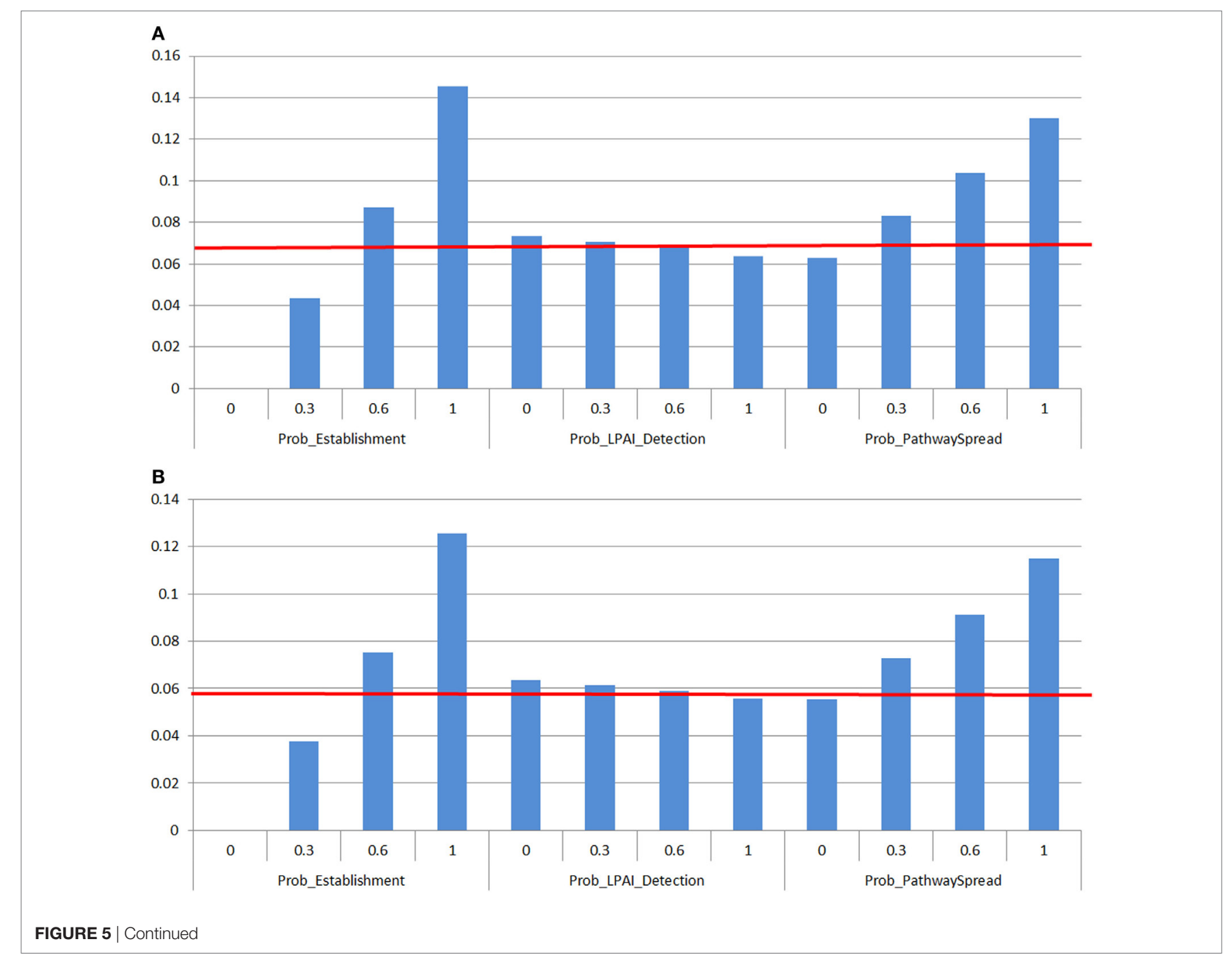




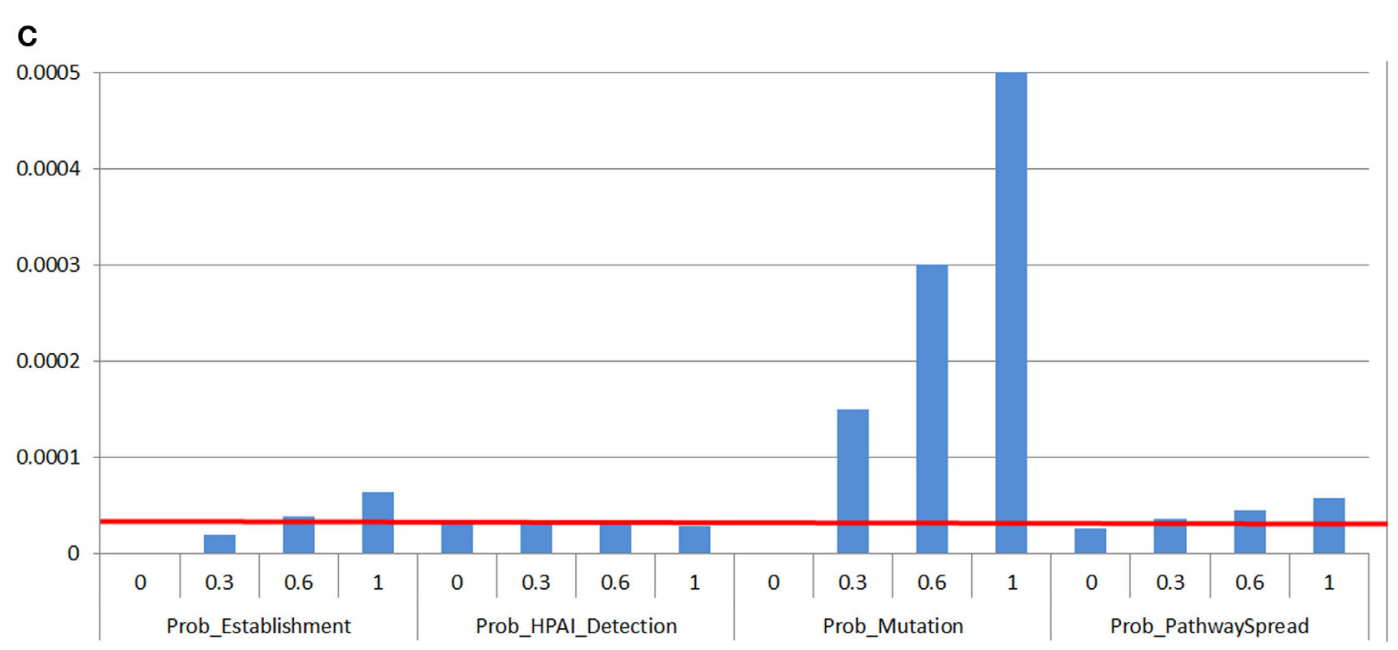

D

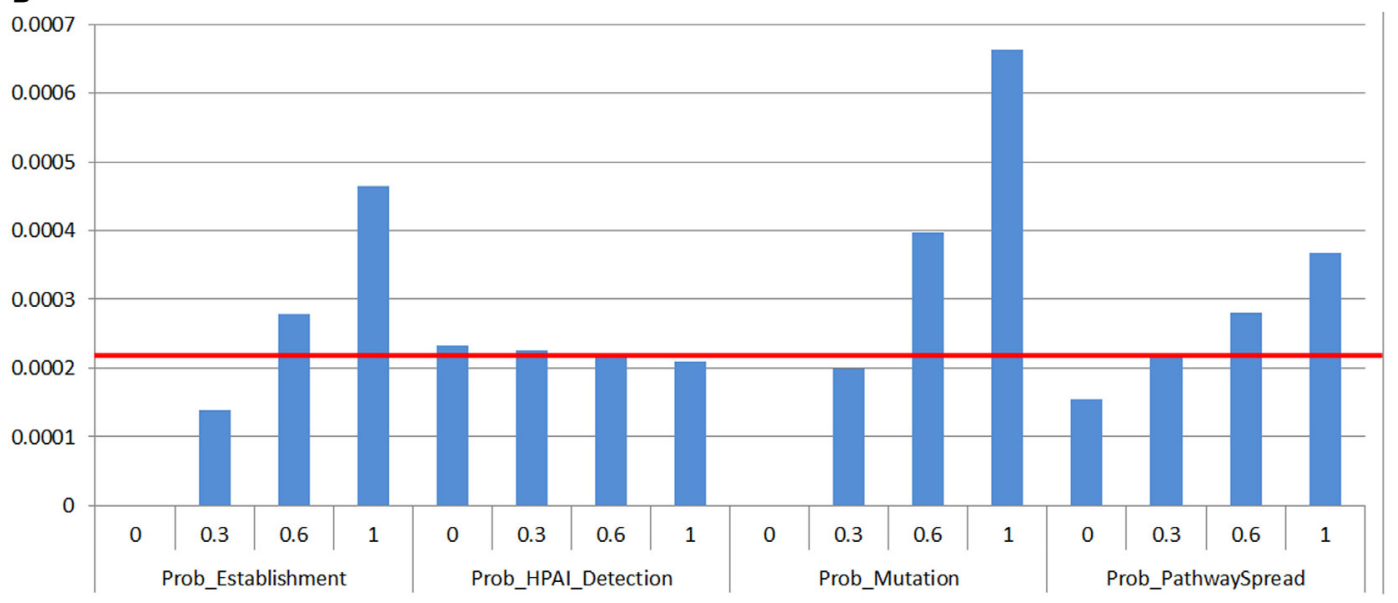

FIGURE 5 | Results of the sensitivity analysis on the spread assessment depicting the change in probability (Y-axis) on the median overall probability of lowpathogenic avian influenza (LPAl) or high-pathogenic avian influenza (HPAl) spread (horizontal line) between sheds on a commercial poultry farm and between commercial poultry farms after exposure of one chicken to low-pathogenic avian influenza (LPAl) virus from wild birds in Australia with changes of certain input variables listed in Table 1 (X-axis). Results were obtained from a simulation of 1,000 iterations using @Risk's Advanced Sensitivity Analysis. The outcomes were similar in proportional increase in value among all farm types so only one example of a meat chicken or layer farm type per LPAI (A,B) or HPAl (C,D) spread between sheds and farms was used. (A) Sensitivity analysis on input parameters affecting the probability of LPAl spread between sheds and farms on free range meat chicken farm types. (B) Sensitivity analysis on input parameters affecting the probability of LPAI spread between sheds and farms on free range layer farm types.

(C) Sensitivity analysis on input parameters affecting the probability of HPAl spread between sheds and farms on barn meat chicken farm types. (D) Sensitivity analysis on input parameters affecting the probability of HPAI spread between sheds and farms on cage layer farm types. Prob_Establishment, Probability that the H5/H7 LPAl subtype will establish within the flock from one infected chicken; Prob_Mutation, Probability that LPAl established within the flock will mutate to HPAl; Prob_LPAI_Detection, Probability that the farmer will detect and report disease to appropriate officials during LPAl establishment; Prob_HPAI_Detection, Probability that HPAl will produce clinical signs with the assumption that the probability of detection is extremely high; Prob_PathwaySpread, Probability of any one of the spread pathways identified, with consideration of the complementary changes for all other spread pathways that will result given sum of the probabilities of all pathways occurring equaled one.

represented changing the probability of any one spread pathway and the complementary changes to the probabilities of the other spread pathways. Increasing any spread pathway to $100 \%$, which results in $0 \%$ probability of all other spread pathways, resulted in approximate doubling of the overall median probability of either LPAI or HPAI spread. This means if the probability of any pathway is certain to occur, and all other pathways are certain not to occur, the probability of either LPAI or HPAI spread is approximately doubled. In reality all other spread pathways will have a probability greater than zero of occurring. It is therefore expected that in a model where such pathways are not complementary to each other, the cumulative effect of increasing the probabilities of each individual pathway will result in greater than doubling the overall probability of LPAI or HPAI spread. The spread pathways are therefore significant influential parameters on the overall probability of spread and are dependent on biosecurity on the farm. Other highly influential parameters in the spread model such as the probability of establishment and mutation are dependent on the mechanisms of the virus and cannot be changed by human intervention. The importance of improving biosecurity on farms in order to reduce the probability of spread is therefore stressed from these results. 


\section{Other Considerations}

These results show the large influence people who are not farm workers but regularly visit the farm have on the probability of spread. Such people include egg pallet and tray collectors and bird pick up crews. Consultation among different industry bodies is important to emphasize shared responsibility and agreement to biosecurity codes and guidelines. Further training for both farm workers and people who visit farms in regard to the importance of biosecurity is always beneficial. The integrated nature of the Australian chicken meat industry by a small number of companies allows for this shared responsibility and relative ease of communication across a range of networks. However, this may well be lacking in the Australian layer sector due to the nature of this industry which has a high level of numerous, privately owned farms (55). As new information arises related to the volume and frequency of spread pathways that occur in the Australian commercial chicken industry, as well as further information on the behavior and mechanisms of the AI virus, these can be used to update the input parameters in the spread scenario tree models.

Detection and reporting was not highly influential in this model as this node simply represented the proportion of farms that would detect or report at a relatively fixed detection threshold. However, this study did indicate that spread between sheds is likely to have already occurred before detection. Other factors related to detection and reporting were not assessed and should be considered for future studies, particularly for high-risk farms. These include those factors assessed in previous modeling studies such as; the impact of lowering the detection threshold, frequent sampling of farms considered high risk, and ensuring prompt action after detection (9-11). Frequent sampling can improve knowledge of LPAI transmission which has been demonstrated to be largely unknown in this study particularly in the Australian context. AI surveillance in poultry in Australia is currently not supported by the industry due to the consequences outlined in the AUSVETPLAN associated with $\mathrm{H} 5$ or H7 detections (56). Performance of surveillance in some form, such as sampling sentinel flocks or poultry at slaughter and processing should be considered for the Australian poultry industry (38).

Given this model considers and follows the probabilities of exposure quantified by Scott et al. (1), the probabilities estimated in this study can be considered representative for the same region as that of Scott et al. (1); the Sydney basin region. Extrapolating these results to other regions, poultry species or non-commercial chicken farms must be done with caution as differences in the probabilities of exposure may exist. However, the framework of this model can be used to aid in the development of similar risk assessment models for these different farms.

\section{CONCLUSION}

This study indicates that the probability of no establishment is the most likely end-point after exposure and infection of LPAI in one chicken. Nodes linked to attributes of the virus, such as the probability of establishment and the probability of mutation, were the most influential factors impacting the probability of
LPAI and HPAI spread, respectively. While these cannot be changed by human intervention, some on-farm actions can be performed to potentially reduce the probability of spread. Biosecurity and cleanliness on farms, with particular attention to equipment and egg trays between sheds and farms, respectively, as these were found as the most likely spread pathways, will reduce the probability of spread. The results of this study and that of the exposure risk assessment in Scott et al. (1) help estimate the overall probability of spread and spread pathways of LPAI and HPAI in Australian commercial chicken enterprises. The results also provide guidance to the Australian commercial chicken industry on the importance of farm workers and people who regularly visit farms in performing biosecurity practices, as this is part of a shared responsibility in safeguarding the industry against AI.

\section{ETHICS STATEMENT}

This study was carried out in accordance with the recommendations of the Human Ethics Committee of the University of Sydney, Australia with written informed consent from all subjects. The protocol was approved by the the Human Ethics Committee of the University of Sydney, Australia (ethics reference number: 2015/252).

\section{AUTHOR CONTRIBUTIONS}

The first author AS was involved with investigation, methodology, writing of the original draft and reviewing and editing. J-AT formed initial conceptualization of the study, and was involved with formal analysis, methodology, project administration, supervision of AS, and reviewing and editing the manuscript. MS was also involved in investigation, methodology, project administration, supervision of AS, and reviewing and editing the manuscript. $\mathrm{BB}$ and $\mathrm{KG}$ were also involved with initial conceptualization of the study, formal analysis, methodology and provided reviewing and editing. $\mathrm{BM}, \mathrm{AB}$ and $\mathrm{PG}$ were involved with conceptualization, project administration funding/support of the study. PG also provided methodology and supervision. BM provided reviewing and editing. MH-J was heavily involved in formal analysis of the results, conceptualization of the study, methodology, project administration, supervision of AS, and reviewing and editing the manuscript.

\section{ACKNOWLEDGMENTS}

The authors extend thanks to the New South Wales Department of Primary Industries, the University of Newcastle and the National Avian Influenza Wild Birds Surveillance Program, which is funded by the Australian Government Department of Agriculture and Water Resources and is administered by Wildlife Health Australia for their collaboration in providing wild bird LPAI virus prevalence data that was used in the exposure model. The New South Wales Department of Primary Industries (NSW DPI) is also appreciated for providing data on actual AI outbreaks that occurred in NSW. Australia, which 
were used for the branching process and spread scenario tree models. Thanks also to the Australian Animal Health Laboratory for providing experimental data on LPAI infections in chickens. The authors also appreciate the experts who attended and completed the expert opinion workshop, of which this data was also used in the spread scenario tree model. Egg and chicken meat companies and producers are also appreciated by the authors for their participation in the farm survey. This research was conducted within the Poultry Cooperative Research Centre (CRC) with support from the CRC and Woolworths Limited. The first author is also a recipient of a Post-graduate Scholarship from the Poultry CRC.

\section{REFERENCES}

1. Scott AB, Toribio J-A, Singh M, Groves P, Barnes B, Glass K, et al. Low pathogenic avian influenza exposure risk assessment in Australian commercial chicken farms. Front Vet Sci (2018) 5:68. doi:10.3389/fvets.2018.00068

2. Spickler AR, Trampel DW, Roth JA. The onset of virus shedding and clinical signs in chickens infected with high-pathogenicity and low-pathogenicity avian influenza viruses. Avian Pathol (2008) 37(6):555-77. doi:10.1080/ 03079450802499118

3. Yao M, Lv J, Huang R, Yang Y, Chai T. Determination of infective dose of H9N2 avian influenza virus in different routes: aerosol, intranasal, and gastrointestinal. Intervirology (2014) 57:369-74. doi:10.1159/000365925

4. FAO EMPRES-i. Disease Events (2016). Available from: http://empres-i.fao. org/eipws3g/. Accessed April 18, 2016.

5. Swayne D, Pantin-Jackwood M. Pathobiology of Avian Influenza Virus Infections in birds and mammals. In: Swayne D, editor. Avian Influenza. Iowa, USA: Blackwell Publishing (2009). p. 87-122.

6. Dorea F, Vieira A, Hofacre C, Waldrip D, Cole D. Stoachastic model of the potential spread of highly pathogenic avian influenza from an infected commercial broiler operation in Georgia. Avian Dis (2010) 53(s1):713-9. doi:10.1637/8706-031609-ResNote.1

7. Ssematimba A, Hagenaars T, de Wit J, Ruiterkamp F, Fabri T, Stegeman J, et al. Avian influenza transmission risks: analysis of biosecurity measures and contact structure in Dutch poultry farming. Prev Vet Med (2013) 109(1-2):106-15. doi:10.1016/j.prevetmed.2012.09.001

8. Scott AB. Avian Influenza Risk Assessment for the Australian Commercial Chicken Industry. PhD Thesis. Sydney: University of Sydney (2018).

9. Comin A, Stegeman A, Marangon S, Klinkenberg D. Evaluating surveillance strategies for the early detection of low pathogenicity avian influenza infections. PLoS One (2012) 7(4):e35956. doi:10.1371/journal.pone.0035956

10. Weaver J, Malladi S, Goldsmith T, Hueston W, Hennessey M, Lee B, et al. Impact of virus strain characteristics on early detection of highly pathogenic avian influenza infection in commercial table-egg layer flocks and implications for outbreak control. Avian Dis (2012) 56(4s1):905-12. doi:10.1637/10189-041012-Reg.1

11. Gonzales J, Boender G, Elbers AR, Stegeman J, de Koeijer A. Risk based surveillance for early detection of low pathogenic avian influenza outbreaks in layer chickens. Prev Vet Med (2014) 117(1):251-9. doi:10.1016/j. prevetmed.2014.08.015

12. Sims LD, Turner AJ. Avian influenza in Australia. In: Swayne D, editor. Avian Influenza. Iowa, USA: Blackwell Publishing (2009). p. 239-50.

13. Brown I, Abolnik C, Gracia-Gracia J, McCullough S, Swayne DE, Cattoli G. High-pathogenicity avian influenza outbreaks since 2008, excluding multi-continental panzootic of $\mathrm{H} 5$ Goose/Guangdong-lineage viruses. In: Swayne D, editor. Animal Influenza. Iowa, USA: Blackwell Publishing (2016). p. $248-70$.

14. Arzey G. Low pathogenic avian influenza in Australia and implications. Proceedings of the Australasian Veterinary Poultry Association (AVPA) Scientific Meeting. Sydney (2013).

15. AVCO. Detection of Low Pathogenic (LP) and Highly Pathogenic (HP) Avian Influenza Virus (AIV) in Flocks of Layers, Breeders and Meat Birds in Australia. Canberra, ACT: Rural and Regional Affairs and Transport

\section{FUNDING}

This study was funded by the Poultry Cooperative Research Centre (CRC) (Sub-project number 1.5.7) and by Woolworths Limited. The funders had no role in study design, data collection and analysis, or preparation of the manuscript.

\section{SUPPLEMENTARY MATERIAL}

The Supplementary Material for this article can be found online at https://www.frontiersin.org/articles/10.3389/fvets.2018.00063/ full\#supplementary-material.

Legislation Committee (2015). Available from: https://www.aph.gov.au/ / media/Committees/rrat_ctte/estimates/bud_1516/ag/answers/QoN62-64. pdf (Accessed: September 1, 2017).

16. Singh M, Ruhnke I, Koning CD, Glatz PC. Demographics and practices of semi-intesnive free-range farming systems in Australia with an outdoor stocking density of $\leq 1500$ hens/hectare. PLoS One (2017) 12(10):e0187057. doi:10.1371/journal.pone.0187057

17. Selleck P, Arzey G, Kirkland P, Reece R, Gould A, Daniels P, et al. An outbreak of highly pathogenic avian influenza in Australia in 1997 caused by an H7N4 virus. Avian Dis (2002) 47:806-11. doi:10.1637/0005-2086-47.s3.806

18. Roth I. Avian influenza outbreak in young - a synopsis. Proceedings of the Australasian Veterinary Poultry Association (AVPA) Scientific Meeting. Gold Coast, Qld (2014).

19. OIE. Chapter 2.1. Import Risk Analysis. In: OIE, editor. Terrestrial Animal Health Code. 20th ed. Paris: OIE (2011). Available from: https://www.oie.int/ doc/ged/D10905.PDF (Accessed: June 15, 2017).

20. Martin P, Cameron A, Greiner M. Demonstrating freedom from disease using multiple complex data sources 1: a new methodology based on scenario trees. Prev Vet Med (2007) 79:71-97. doi:10.1016/j.prevetmed.2006.09.007

21. Scott AB, Singh M, Groves P, Hernandez-Jover M, Barnes B, Moloney KGB, et al. Comparisons of management practices and farm design on Australian commercial layer and meat chicken farms: cage, barn and free range. PLoS One (2017) 12(11):e0188505. doi:10.1371/journal.pone.0188505

22. Singh M, Toribio J-A, Scott A, Groves P, Barnes B, Glass K, et al. Assessing the probability of introduction and spread of Avian Influenza (AI) virus in commercial Australian poultry operations using an expert opinion elicitation. PLoS One (2018) 13(3):e0193730. doi:10.1371/journal.pone.0193730

23. Selleck P. AAHL AI Experiments Internal Report. Geelong: AAHL (2015).

24. Webster R, Yakhno M, Hinshaw V, Bean W, Murti KG. Intestinal influenza: replication and characterisation of influenza viruses in ducks. Virology (1976) 84:268-78. doi:10.1016/0042-6822(78)90247-7

25. Jonges M, Leuken JV, Wouters I, Koch G, Meijer A, Koopmans M. Windmediated spread of low pathogenic avian influenza virus into the environment during outbreaks at commercial poultry farms. PLoS One (2015) 10(5):e0125401. doi:10.1371/journal.pone.0125401

26. Barnes B, Glass K. Modelling Low Pathogenic Avian Influenza Introduction into the Commercial Poultry Industry (2018).

27. Spackman E, Gelb J, Preskenis LA, Ladman BS, Pope CR, Pantin-Jackwood MJ, et al. The pathogenesis of low pathogenicity $\mathrm{H} 7$ avian influenza viruses in chickens, ducks and turkeys. Virol J (2010) 7:331. doi:10.1186/1743-422X-7-331

28. Mo I, Brugh M, Fletcher O, Rowland G, Swayne D. Comparative pathology of chickens experimentally inoculated with avian influenza viruses of low and high pathogenicity. Avian Dis (1997) 41:125-36. doi:10.2307/1592452

29. Jones YL, Swayne DE. Comparative pathobiology of low and high pathogenicity H7N2 Chilean avian influenza viruses in chickens. Avian Dis (2004) 48:119-28. doi: 10.1637/7080

30. Swayne DE. Avian Influenza. Iowa, USA: Blackwell Publishing (2008).

31. Achenbach JE, Bowen RA. Transmission of avian influenza A viruses among species in an artificial barnyard. PLoS One (2011) 6(3):e17643. doi:10.1371/ journal.pone.0017643

32. Nielsen AA, Sovgard H, Stockmarr A, Handberg KJ, Jorgensen PH. Persistence of low-pathogenic avian influenza H5N7 and H7N1 subtypes in house flies 
(Diptera: Muscidae). J Med Entomol (2011) 48(3):608-14. doi:10.1603/ ME11017

33. Tiwari A, Patnayak DP, Chander Y, Parsad M, Goyal SM. Survival of two avian respiratory viruses on porous and nonporous surfaces. Avian Dis (2006) 50(2):284-7. doi:10.1637/7453-101205R.1

34. Wood J, Choi Y, Chappie D, Rogers J, Kaye J. Environmental persistence of a highly pathogenic avian influenza (H5N1) virus. Environ Sci Technol (2010) 44:7515-20. doi:10.1021/es1016153

35. Sawabe K, Tanabayashi K, Hotta A, Hoshino K, Isawa H, Sasaki T, et al. Survival of Avian H5N1 influenza A viruses in Calliphora nigribarbis (Diptera: Calliphoridae). J Med Entomol (2009) 46(4):852-5. doi:10.1603/033.046.0416

36. Nettles VF, Wood JM, Webster RG. Wildlife surveillance associated with an outbreak of lethal H5N2 avian influenza in domestic poultry. Avian Dis (1985) 29(3):733-41. doi:10.2307/1590665

37. McCluskey BJ. In: United States Department of Agriculture (USDA), editor. Epidemiologic and Other Analyses of HPAI-Affected Poultry Flocks: July 15, 2015 Report. Colorado, USA: Animal and Plant Health Inspection Services (APHIS) (2015).

38. East I, Ainsworth C, Warner S, Dunowska M, Azuolas J. Seroconversion to avian influenza virus in free-range chickens in the Riverland region of Victoria. Aust Vet J (2010) 88(8):290-3. doi:10.1111/j.1751-0813.2010.00601.x

39. CDC. Highly Pathogenic Avian Influenza A (H5N1) in Birds and Other Animals. Atlanta: CDC (2015). Available from: https://www.cdc.gov/flu/ avianflu/h5n1-animals.htm (Accessed: July 5, 2017).

40. AHA. Disease Strategy: Avian Influenza (Version 3.4). Australian Veterinary Emergency Plan (AUSVETPLAN). 3rd ed. Canberra, ACT: Primary Industries Ministerial Council (2011).

41. DEFRA. Notifiable Avian Disease Control Strategy for Great Britain. Crown: DEFRA (2012).

42. USDA. Highly Pathogenic Avian Influenza Response Plan - The Red Book. Maryland, USA: Foreign Animal Disease Preparedness \& Response Plan (2017).

43. Siettou C. Avian Influenza: outbreaks and the impact on UK consumer demand for poultry. 90th Annual Conference of the Agricultural Economics Society. Warwick, UK (2016).

44. Greene JL. Update on the Highly-Pathogenic Avian Influenza Outbreak of 20142015. Washington, DC: Congressional Research Service (2015). Available from: https://fas.org/sgp/crs/misc/R44114.pdf (Accessed: July 10, 2017).

45. Veterinary Record. Highly pathogenic avian influenza spreads in the USA. Veterinary Record (2015) 176:505. doi:10.1136/vr.h2582

46. DAF. Outbreaks of Avian Influenza. Brisbane: Queensland Government (2013).

47. New South Wales Government. Highly Pathogenic Avian Influenza (H7N7) Maitland-Control and Eradication (2012). Available from: http://archive.dpi. nsw.gov.au/content/media-releases/2012/avian-influenza-hunter (Accessed: April 5, 2015).
48. Australian Government. High Pathogenic Avian Influenza - Young, NSW. Canberra, ACT: Commonwealth of Australia (2013). Available from: http:// www.agriculture.gov.au/about/media-centre/communiques/high-pathogenic-avian-influenza-a-young-nsw (Accessed: April 5, 2015).

49. Hamilton S. Simulating the Transmission and Control of Highly Pathogenic Avian Influenza Epidemics in the Australian Poultry Industries? PhD Thesis, University of Sydney, Sydney (2011).

50. Gonzales J, Goot JV, Stegeman J, Elbers A, Koch G. Transmission between chickens of an H7N1 low pathogenic avian influenza virus isolated during the epidemic of 1999 in Italy. Vet Microbiol (2011) 152(1-2):187-90. doi:10.1016/j. vetmic.2011.04.022

51. Richard M, Fouchier R, Monne I, Kuiken T. Mechanisms and Risk Factors for Mutation from Low to Highly Pathogenic Avian Influenza Virus. Parma: European Food Safety Authority (2017).

52. Bouma A, Claassen I, Natih K, Klinkenberg D, Donnelly CA, Koch G, et al. Estimation of transmission parameters of $\mathrm{H} 5 \mathrm{~N} 1$ avian influenza virus in chickens. PLoS Pathog (2009) 5:e1000281. doi:10.1371/journal.ppat.1000281

53. Gonzales J, Elbers A, Bouma A, Koch G, Wit JD, Stegeman J. Transmission characteristics of low pathogenic avian influenza virus of H7N7 and H5N7 subtypes in layer chickens. Vet Microbiol (2012) 155(2-4):207-13. doi:10.1016/j. vetmic.2011.09.016

54. Hamilton S, East I, Toribio J-A, Garner M. Are the Australian poultry industries vulnerable to large outbreaks of highly pathogenic avian influenza? Aust Vet J (2009) 87:165-74. doi:10.1111/j.1751-0813.2009.00423.x

55. Scott P, Turner A, Bibby S, Chamings A. In: Department of Agriculture Fisheries and Forestry DAFF, editor. Structure and Dynamics of Australia's Commercial Poultry and Ratite Industries. Moonee Ponds, Victoria: Scolexia Animal and Avian Health Consultancy (2009).

56. Animal Health Australia. Disease strategy: Avian influenza (Version 3.4). Australian Veterinary Emergency Plan (AUSVETPLAN). 3rd ed. Canberra, ACT: Primary Industries Ministerial Council (2011). Available from: http:// www.animalhealthaustralia.com.au/programs/emergency-animal-disease-preparedness/ausvetplan/ (Accessed: April 10, 2015).

Conflict of Interest Statement: The authors declare that the research was conducted in the absence of any commercial or financial relationships that could be construed as a potential conflict of interest.

Copyright (c) 2018 Scott, Toribio, Singh, Groves, Barnes, Glass, Moloney, Black and Hernandez-Jover. This is an open-access article distributed under the terms of the Creative Commons Attribution License (CC BY). The use, distribution or reproduction in other forums is permitted, provided the original author(s) and the copyright owner are credited and that the original publication in this journal is cited, in accordance with accepted academic practice. No use, distribution or reproduction is permitted which does not comply with these terms. 